\title{
Vibration measurements by tracking laser doppler vibrometer on automotive components
}

\author{
P. Castellini \\ Università degli Studi di Ancona, Dipartimento di Meccanica via Brecce Bianche, 60131 Ancona, Italy
}

\begin{abstract}
This paper describes the application of a Tracking Laser Doppler Vibrometer (TLDV) to the measurement of vibration of some typical automotive component.

After a presentation and discussion of the measurement technique, the attention is focused on the development of specific version optimised for each application.

The first component analysed is the sidewall of a tire during its rotation in a typical drum test-bench.

An optimised version of the TLDV was developed for the specific application adding a trajectory assessment tool based on image analysis, in order to fulfil the accuracy specifications imposed by tire manufacturer.

The second automotive component is a timing belt.

This application presents problems related to the high-speed linear motion and to data processing for noise rejection.

The third application is on windscreen wipers. In this case the tracking approach fully demonstrate his capabilities, representing the only technique able to give information in the time domain on the dynamic behaviour of the rubber blade in operative conditions.

All the application shows as the TLDV allows to obtain realistic results on the dynamic characteristics under simulated operative conditions.

A Lagrangian approach was adopted: data were acquired with the target in continuously changing conditions and that impose a not traditional approach on LDV data such as a joint time-frequency analysis.
\end{abstract}

\section{Introduction}

Nowadays the study of vibrations of automotive components is of increasing interest in the automotive industry. In fact, the noise emitted by (and the comfort related to) vibrations is becoming a critical point the impact of cars on the environment.

New standard rules on noise reduction determine a strong pressure on designers. Some components, such as that build in rubber or similar materials, are particularly interesting for large possibilities that they offer for vibrational improvement.

Tires, timing belts and windscreen wipers have structures really complicated both in terms of materials behaviour, both in terms of geometry and constraint interactions.
In fact, the particular, and unchangeable, position of tyres or wipers in a car does not allow the application of noise reduction panels or other attenuation element. Consequently it is necessary to work directly on the mechanism of vibration and noise production, which is depending by the complex interaction of the friction. Similarly the timing belts, because of the interaction of the teeth, that is also related to reliability.

These are the main reasons why, in order to be able to evaluate the mechanisms of vibration and noise emission, the present trend in components production, is the development of very sophisticated solutions in particular for what concerns experimental procedures for parameters assessment.

Numerical and experimental simulations are not completely satisfying and the experimental analysis in operative conditions appear as not replaceable. 
In addition, for this kind of tests traditional techniques demonstrated large limits. In fact contact sensors, like accelerometers, are too much invasive for operative tests.

On the other hand, field techniques, as holography, are not able to give a good representation of time histories of vibrations, and therefore to give information about the frequency and damping behaviour of the tyre.

The Laser Doppler Vibrometry [1], and in particular the Tracking Laser Doppler Vibrometer [2-4], is a technique that also allows measurements in operative conditions.

This paper describes the application of a Tracking Laser Doppler Vibrometer to the measurement of vibration of some automotive component in motion during his work.

\section{The tracking laser doppler vibrometer: The concept design}

The Tracking Laser Doppler Vibrometer is a technique that allows measurements of vibration behaviour of a component, which presents large movements during its vibration. TLDV extends the capability of a laser Doppler vibrometer to analyse the behaviour of a structure also in operative conditions. In fact, by following each point of interest with the measurement laser spot it is possible to measure its vibration time history during the whole trajectory. The metrological performances are similar to that of a standard laser vibrometer, in particular in terms of high sensitivity and resolution, absence of intrusiveness and large bandwidth. In addition, the tracking allows to eliminate any relative velocity between the laser spot and the surface, thus drastically reducing the optical noise (such as speckle noise) and improving the Signal-to-Noise ratio (SNR).

Depending of the specific application different version of TLDV was developed.

In fact the sensor that generate the signal of the position of the target, which is one of the most important component of the TLDV, must be optimised depending of the specific application.

In this work three version of the system was presented:

1. Encoder-based for rotational motion, applied for sidewall tire and simple wiper;

2. Encoder-based for linear motion, applied for timing belt and tire treads;

3. Wire potentiometer for arbitrary motion, applied for complex wipers.
Differences are on the sensor used for position assessment but also on tracking strategy and algorithms, depending by the relationship between the assessed position and the actual target motion.

\section{Measurements on tires}

The TLDV can be useful applied for the measurement of the vibration pattern both of tire sidewall and of tire treads.

In fact, the ability to track moving object not only improves the SNR for measurement on non-cooperative surfaces, but also allows to directly evaluate the behaviour of tire parts at the exit of the contact area, and to assess the damping characteristics.

Tests where performed during a drum test, which simulate the conditions of contact between the tire and the road.

The measurement system is based on a Scanning Laser Doppler Vibrometer (SLDV), in which mirrors are controlled in order to obtain a tracking synchronised with the motion of a rotating structure.

The system uses an encoder on the rotor as feed-back sensor; in practice the laser beam is moved at the angular location specified by the encoder reading, which is used as the set-point of the two PID controllers which drive the mirrors. The position of the laser beam can be obtained from the signals coming from the position sensors mounted on the mirrors. The encoder issues two pulse trains, each with 2880 pulses per revolution. The D/A converter of the board employs such signal in order to generate analogue voltages to drive the LDV mirrors directly. The same signal can be used to synchronise data acquisition with angular position, or the vibration signals may be acquired on an independent time base. The $1 / \mathrm{rev}$ pulse signal from the encoder is used for the tracking trigger, so that each acquisition starts at the same angular position of the rotating object.

The encoder determinates the position accuracy. An angular uncertainty of about $0.125^{\circ}$, with a resolution better than $1 \mathrm{~mm}$, has been achieved [3].

The tracking algorithm is the most important part of the tracking software. The first objective in its development was to limit calculations performed by the CPU of the PC as much as possible; this is possible if data are processed by the A/D conversion board, allowing the CPU to carry out other operations, such as signal processing.

Such results have been obtained by using the buffered signal generation of the AT-MIO 16 E 10 board. The 


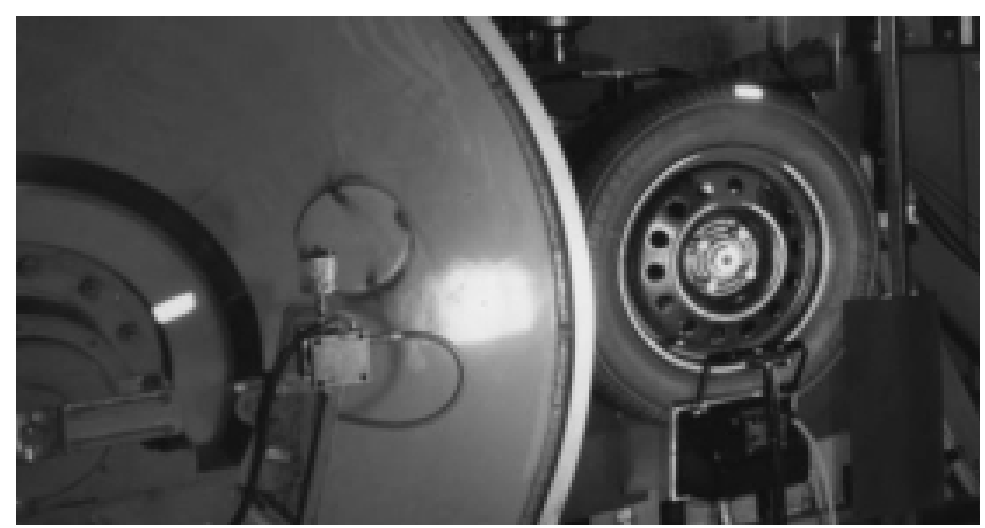

Fig. 1. The high speed uniformity machine at the firestone-bridgestone TCE.

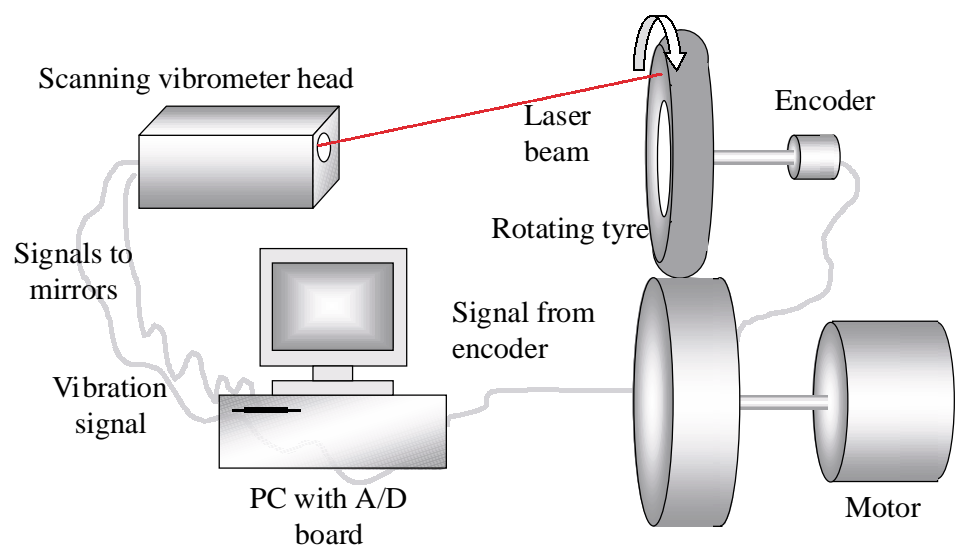

Fig. 2. Basic arrangement of the test bench.

same DAQ board can manage the data signal acquisition.

The tracking and acquisition management software has been created in LabVIEW ${ }^{\circledR}$. An easy-to-use graphic user interface (GUI) has been developed to facilitate the measurement procedure. The software has a virtual front panel, which appears like that of a standard Scanning Laser Doppler Vibrometer.

\subsection{The test bench for tires measurements}

The tests were performed at the Technical Centre Europe (TCE) of Bridgestone-Firestone, in Rome (Italy).

The drum test was performed in the High-Speed Uniformity Machine at TCE, which represent the typical bench used by tire manufacturers to evaluate tire parameters under rolling conditions. An image of the bench is shown in Fig. 1.

This is a rotating drum bench in which a large drum (1.78 $\mathrm{m}$ of diameter) is used to simulate the road. The tyre is rolling on the drum surface and the velocities and the distance between the two shafts and the six components of the load are continuously measured and controlled.

The bench is able to test tyres at a velocity up to $240 \mathrm{~km} / \mathrm{h}$ and at a load up to $1000 \mathrm{~kg}$.

The TLDV set-up for this kind of test is shown in Fig. 2.

\subsection{Trajectory assessment}

In first approximation the trajectory of each point of the sidewall of a tire can be considered circular, but in correspondence to the contact area the trajectory of sidewall points is deformed, as shown in Fig. 3.

In order to perform a correct tracking it is necessary to assess the quasi-circular trajectory of target points.

The trajectory of a point of the tyre's sidewall is not simply predictable, depending on several parameters, 


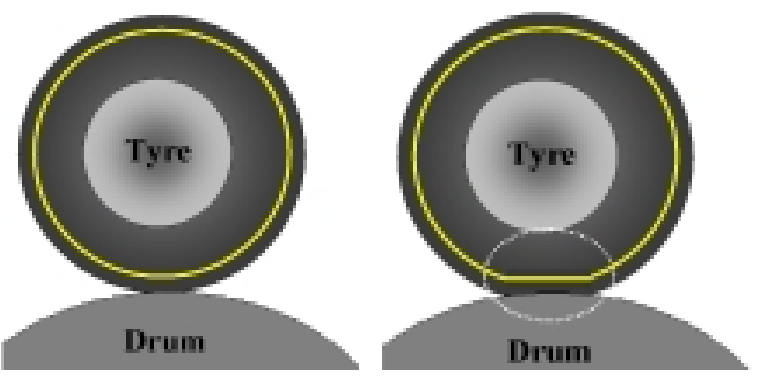

Fig. 3. Theoretical and actual trajectory.

like radial position, speed, load and, finally, the tyre features.

It is then necessary to have a complete system, able to rapidly measure the trajectory of the specific target point and the vibration in tracking conditions, while the test conditions are stationary.

Therefore we designed and realised an additional system, based on image analysis, for the fast trajectory evaluation.

As shown in Fig. 4, the system is based on a highresolution camera, which observe two small retroreflective markers, one glued on the tyre and the other on the wheel.

The software individuates both the trajectories and performs a perspective correction by comparing the rim trajectory (in any case circular) with the trajectory of the marker on the tire (depending on speed, load, tyre pressure etc.).

The spatial resolution of the technique is basically determined by the resolution of the camera and, applied to a typical automotive tyre, it is better than $0.3 \mathrm{~mm}$, without considering the benefit of the sub-pixel interpolation applied on image processing algorithm.

The system has been tuned-up and tested on the High Speed Uniformity Machine in Bridgestone-Firestone Technical Centre Europe.

In Fig. 5 an example of the results is shown. The evaluated average trajectory presents the effect of the contact of the tyre with the drum and some irregularities due to vibrations of the tyre, that must be filtered out.

\subsection{Results and data processing}

In Fig. 6 the comparison between vibration timehistories obtained with the tracking system operating on circular and on evaluated trajectory is shown.

In practice, the tracking of a circular trajectory provides not accurate measure in correspondence to the contact area. In fact in that area the laser beam is measuring on different points with a different veloci- ties. The velocity values are overestimated or underestimated depending of the radial position of the analysed point and of the tire deformation (higher in the case of Fig. 8). Of course, out of the contact zone, the trajectories, and then the measured vibration velocities, are quite the same.

Obtained data can be processed with different approaches and in different domains.

\subsubsection{Time domain}

In Fig. 7 there are some results obtained analysing a 195/65 R15 tyre at $40 \mathrm{~km} / \mathrm{h}$. In particular time histories of the vibration of four points at different radial positions, in the same conditions of load and pressure, are shown.

Generally each sidewall point vibrates with a null average velocity when far from the ground; in fact its conditions are almost identically to a suspended turning tyre. When the point approaches the ground, tyre carcass is deformed: the tread becomes flat and the sidewall curvature increases. For this reason sidewall points move outward in correspondence of the leading edge of the contact area, they reach their maximum displacement near the centre of the footprint (where velocity is zero) and move inward in the trailing edge when the carcass recover its original shape. This explains the quick velocity fluctuation near the half of the revolution time: maximum velocity is at almost half sidewall height.

Changing the structure stiffness, as example testing different tire size or changing the internal pressure, corresponding variation of velocity peaks can be appreciated.

Starting from the measured data, a waterfall-like graphic, showing the vibration velocity time histories for different radial positions, can be constructed (Fig. 8). Rows represent velocity time histories at a specific radial position, the wheel on the top and the ground on the bottom, while columns show the instantaneous velocity at a specific time (or angle of rotation) of a radial profile of the tire. Different grey levels represent vibration velocity amplitudes. At the right (i.e. after) of the contact area the damped oscillations are shown as typical waves propagating from the middle of the graph.

\subsubsection{Frequency domain}

Frequency analysis of time history allows finding several natural frequencies of the tyre. The frequencies below $50 \mathrm{~Hz}$ are due to the large sidewall displacement 

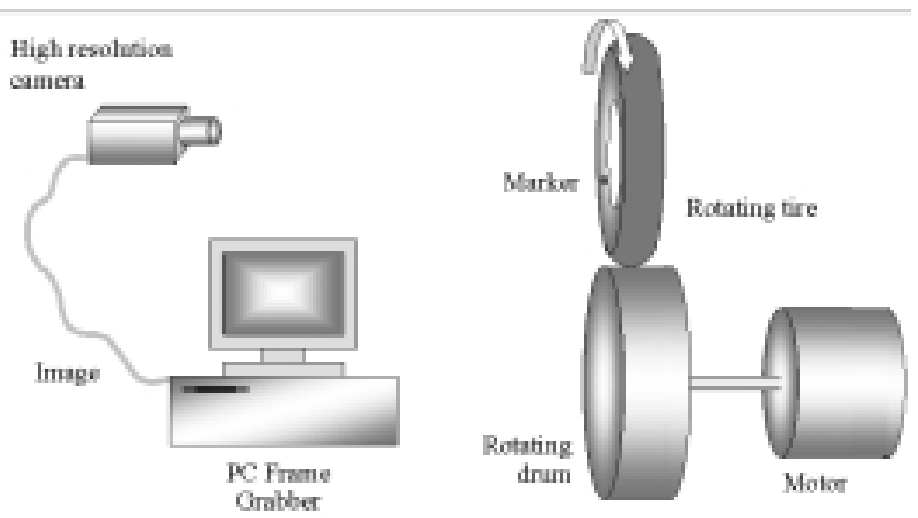

Fig. 4. Tracking assessment system scheme.

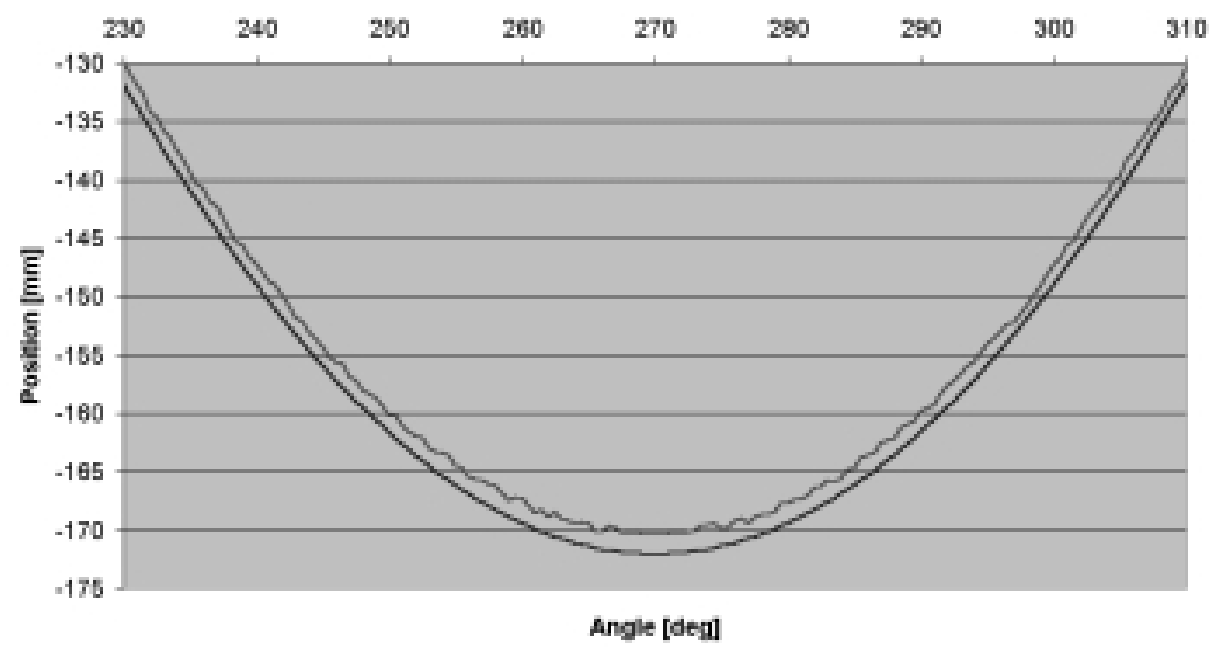

Fig. 5. Comparison between evaluated (light) and circular (dark) trajectories.

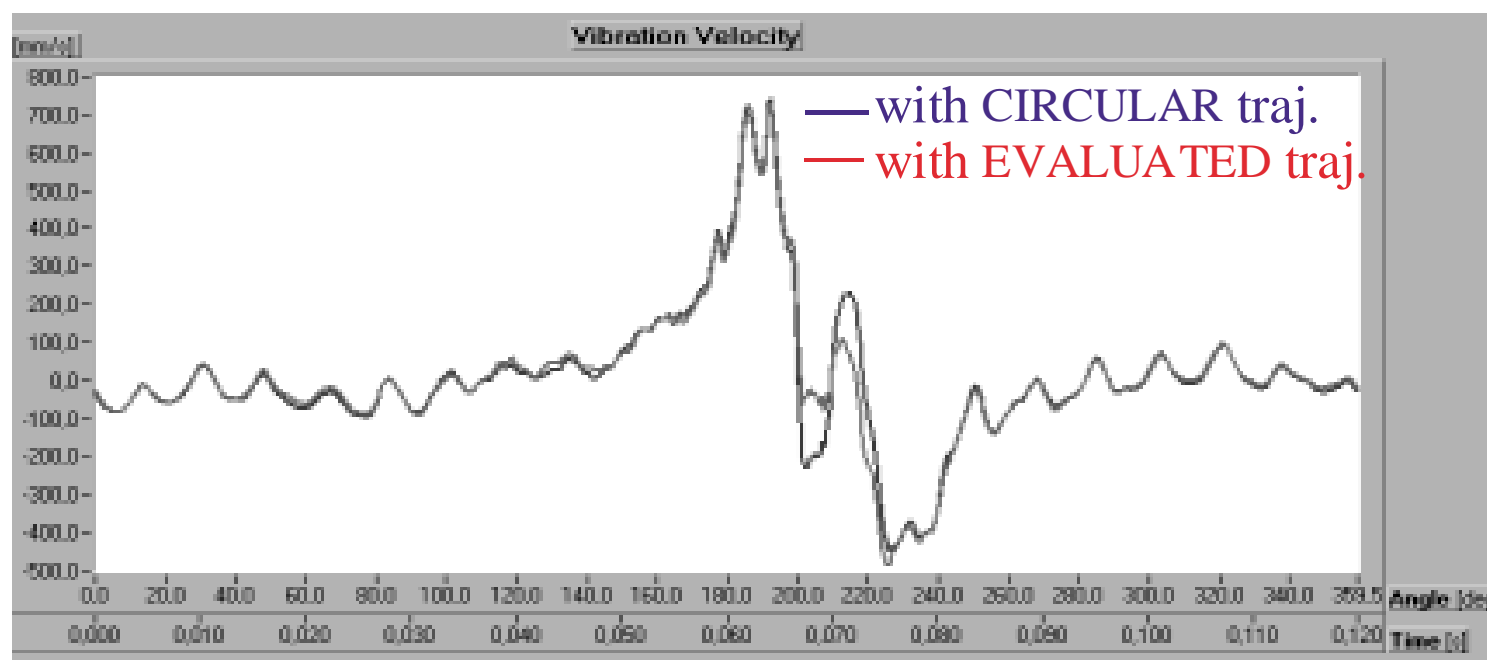

Fig. 6. Comparison between vibration time histories obtained with evaluated and circular trajectories. 

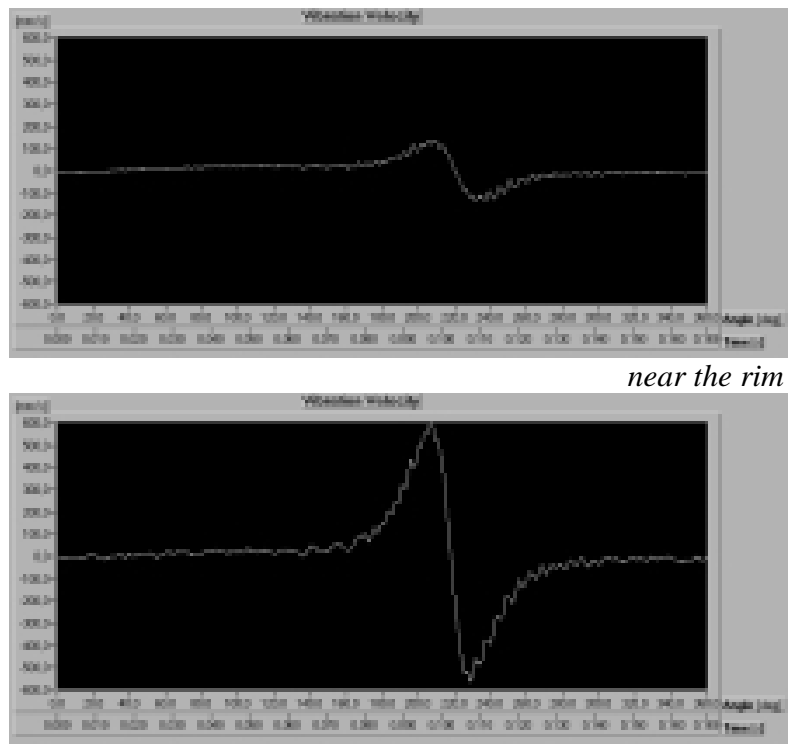

$66 \%$ from the rim to the drum

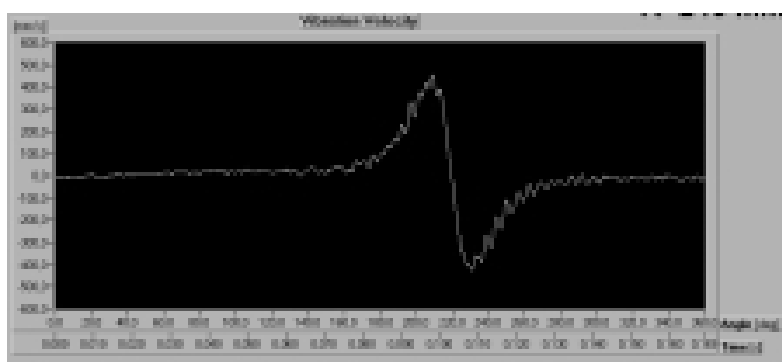

$33 \%$ from the rim to the drum

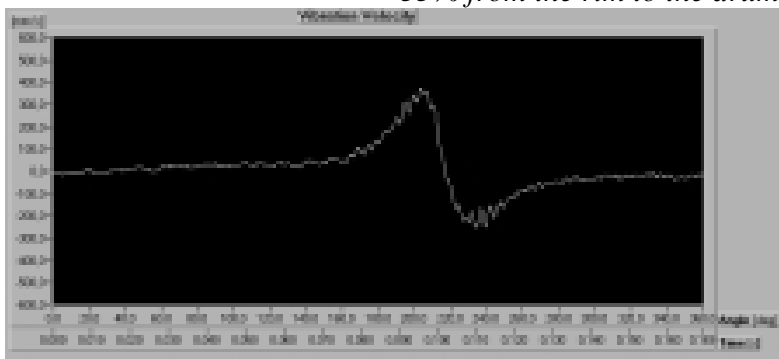

near the drum

Fig. 7. Time histories of the vibration velocity of points at different radial positions for a $195 / 65 \mathrm{R} 15$ tyre at $40 \mathrm{~km} / \mathrm{h}$.

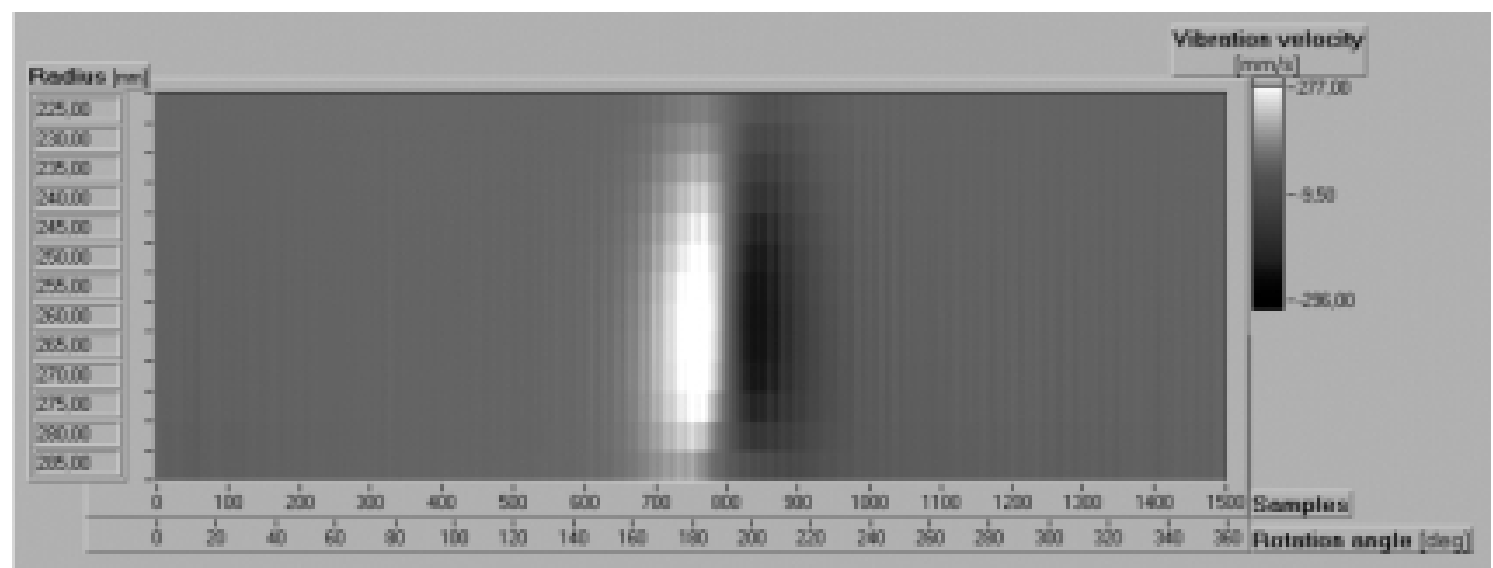

Fig. 8. Map of the vibration velocity time histories of the tire rotating at velocity $40 \mathrm{~km} / \mathrm{h}$.

produced when the measurement point approaches the contact area.

Observing, as example, the spectrum obtained with the 175/80R15 tyre (Fig. 9) we can note that a correct understanding of behaviour is not easy, also for the broadening of vibration energy.

However it can be found the peaks corresponding to $70 \mathrm{~Hz}$ and $135 \mathrm{~Hz}$ probably related to the first two natural modes; peaks are also present at $245 \mathrm{~Hz}$ and $298 \mathrm{~Hz}$ : it is not trivial to say if the first peak depends on the cavity resonance, theoretically at $206 \mathrm{~Hz}$.

No large peaks are present above $400 \mathrm{~Hz}$, confirming that tyre structure is not very stiff. It could be sup- posed that the low stiffness involves non-linear effects in the modes dynamics, producing the previous described broadband spectrum. However, this spectrum shows a high modal density typical of rolling tyres: this means that peaks due to structure resonance are very close and sometimes partially overlapped.

Comparing with the other points, the amplitude of natural frequencies changes with the measurement point, depending on the mode shape.

Even if this analysis gives interesting information about the natural frequencies of the tyre, it is not possible to relate each one to the mode shape, in order to recognise exactly to which mode it can be related. 


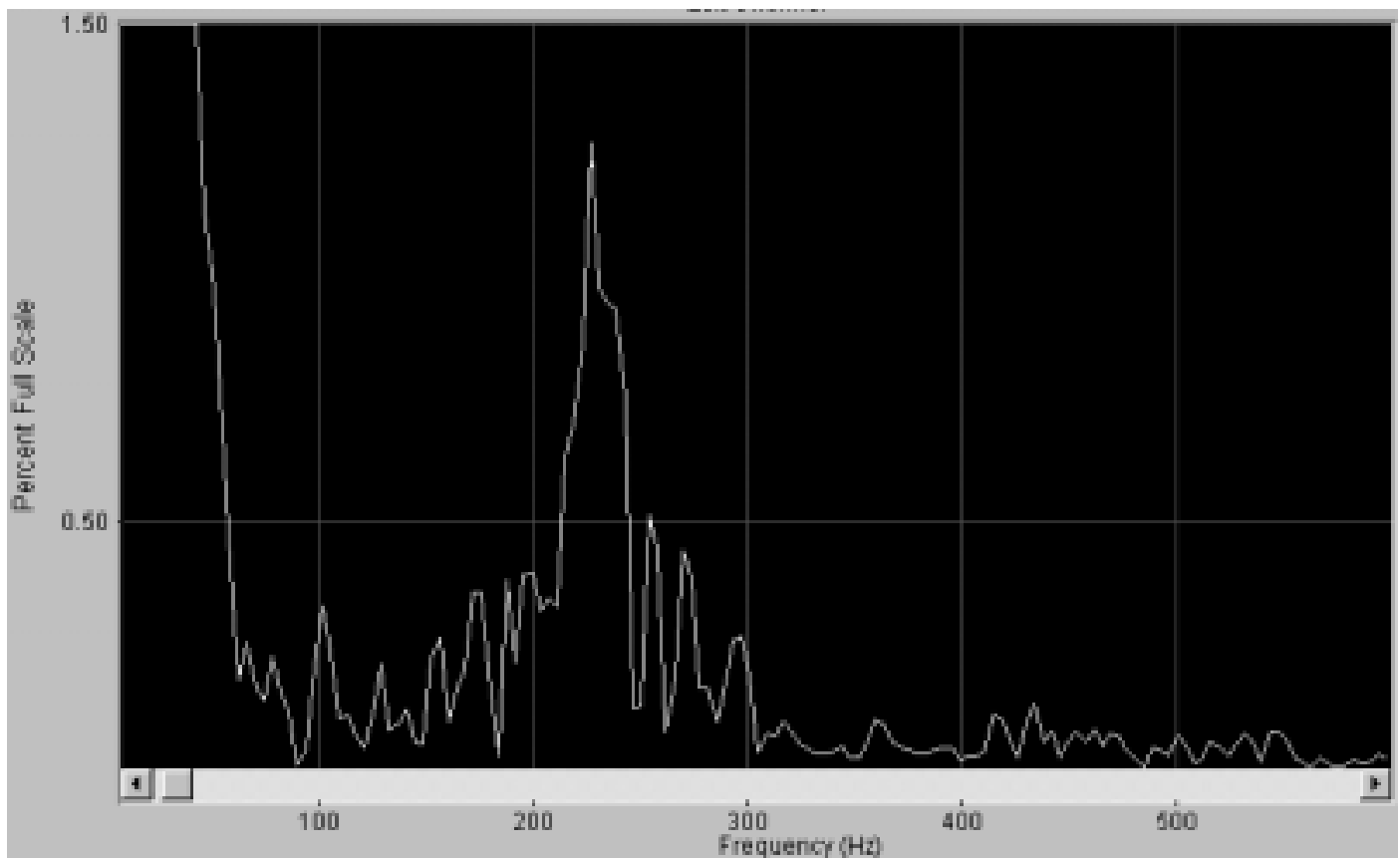

Fig. 9. Measured spectrum for the $195 / 65 \mathrm{R} 15$ tyre at $20 \mathrm{~km} / \mathrm{h}$ with $265 \mathrm{~mm}$ radial position.

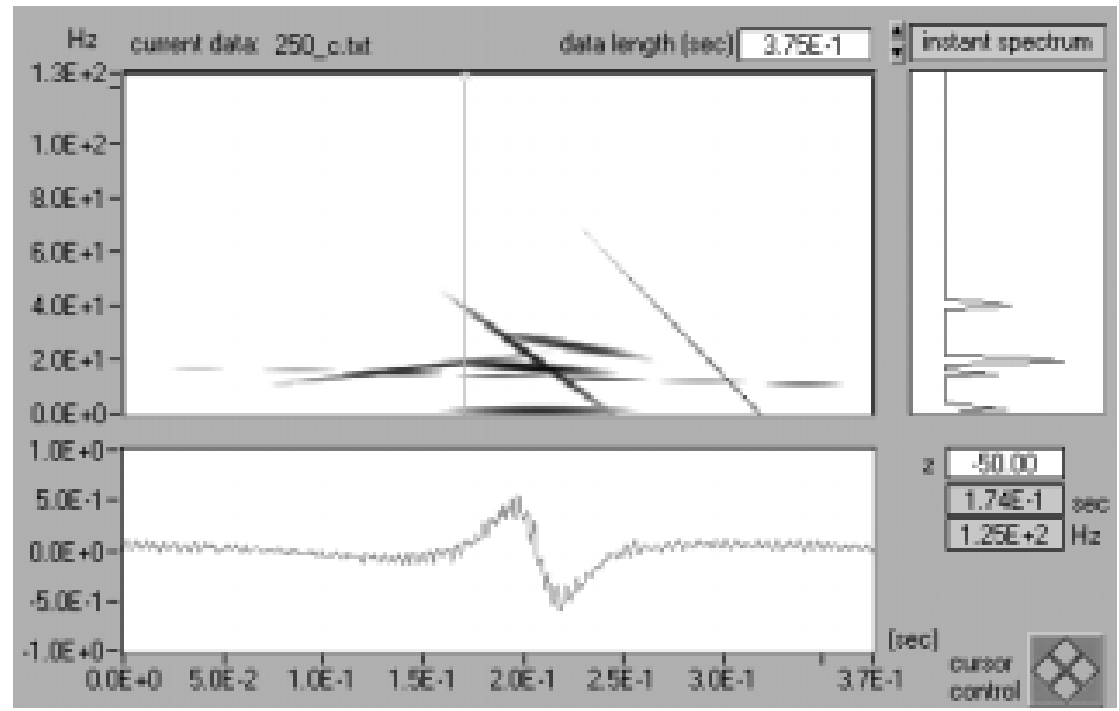

Fig. 10. Example of JTFA result on the TLDV signal (Adaptive spectrogram).

In conclusion, the deep interpretation of the meaning of each peak is not possible, because of the average effect of the spectrum performed on non-stationary data. Peaks on the spectrum could be just due to the shift of an oscillation frequency.

On the other hand transient phenomena, that interest just few samples, could have a low weight on the spectrum and therefore disappears. Of course it is pos- sible to recognise some contribution, but loosing further information, and substantially reducing the spatial resolution of the analysis.

\subsubsection{Time-scale domain}

In order to overcome this drawback one possibility is to divide the signal (windowing) into several blocks that can be overlapped and then applied the Fourier trans- 
form to every individual block of data to indicate the frequency contents of each. These technique, known as the Short-Time Fourier Transform (STFT) maps a signal into a two dimensional function of time and frequency and roughly reflects how frequency contents change over time. The STFT represents a sort of compromise between time and frequency based views of a signal but the precision that can be obtained is limited by the Heisenberg uncertainty relation, i.e. it is not possible to obtain high resolution in both the time and frequency domain. Another disadvantage is that once a particular size for the time window has been chosen, that window remains the same for all frequencies. Many signals require a more flexible approach allowing to vary the window size to determine more accurately either time or frequency. In order to extend the capabilities of Joint Time-Frequency Analysis (JTFA) several others decomposition algorithms (Wigner-Ville Distribution, Choi-Williams Distribution, Cone Shaped Distribution, Gabor Spectrogram, Adaptive Spectrogram, Wavelet) have been developed in the last years and many new energy distributions have been proposed.

The TLDV signal of the rolling tire can be processed using different JTFA algorithms, as shown in [5,6]. An example of typical results is shown in Fig. 10.

\section{Measurements on timing belts}

The concept of Tracking Laser Doppler System (TLDV) has been developed, both scanning hardware and control software, for vibration tests on linear motion.

Such an approach is possible whenever the mechanism has a known kinematics; in a 1 degree of freedom mechanism, if a sensor provides information on the position of one link, then the position of any other member of the kinematics chain can be known. Therefore the position of the target point is computed as a function of one measured position and then used to drive the scanning mirrors.

For the measurement of linear motion two approaches are possible.

The first, and the most natural, is possible when a sensor, which gives directly the position of the target, is available, as shown in Figure 11. In this case the tracking strategy is basically the same of the rotational tracking with linear instead of angular coordinates.

The second approach is necessary when direct information of the position is not available, but it is possible to use indirect information of the position of the tar- get, as shown in Fig. 12. In these cases the relationship between measured and actual position of the target must be known through a model of the mechanism kinematics.

In a 1 degree of freedom mechanism, if a sensor provides information on the position of one link, the position of any other member of the kinematics chain can be known. Therefore the position of the target point is computed as a function of one measured position and then used to drive the scanning mirrors.

In the application on timing belts the first approach is practically impossible, being impossible to connect a position sensor directly on the running belt.

In order to measure the actual position of the belt an encoder is installed on the pulley, so that its angular position is measured, as shown in Fig. 13. In practice the laser beam is moved at the angular location specified by the encoder reading, which is used as the set-point of the two PID controllers which drive the scanning mirrors. Actually the control scheme is an open loop control, because no feed-back is available on the actual position achieved by the laser beam.

The encoder signal provides the angular position and the D/A converter of the PC board employs such signal in order to generate analogue voltages to drive the scanning mirrors.

The tracking is therefore based only on the kinematics model of the mechanism which provide information for writing the signal buffer to be sent to mirror controller and which represent the actual position of the target for each position of the pulley. Of course this model must take into account also the position of the vibrometer head respect to the bench.

The TLDV set-up is related to dimensions of the belt and to relative position between the bench and the vibrometer. If the distance between the belt and the TLDV is increased, then the total scan angle is decreased and the angular speed of the rotating mirrors is decreased as well, as shown in Fig. 14. Measurements were taken with TLDV at $3 \mathrm{~m}$ from the belt. In order to simplify the measurement procedure, a software alignment tool was developed which determine the relative position, the trigger conditions and the measurement interval across which the scan occurs.

An important feature of the TLDV, highlighted in the application on timing belt, is the reduced sensitivity to disturbing inputs on the measured signal.

The first effect, already previously discussed, is the virtual elimination of the speckle noise due to the presence of tangential velocity on the target on which outof-plane measurement are performing. 


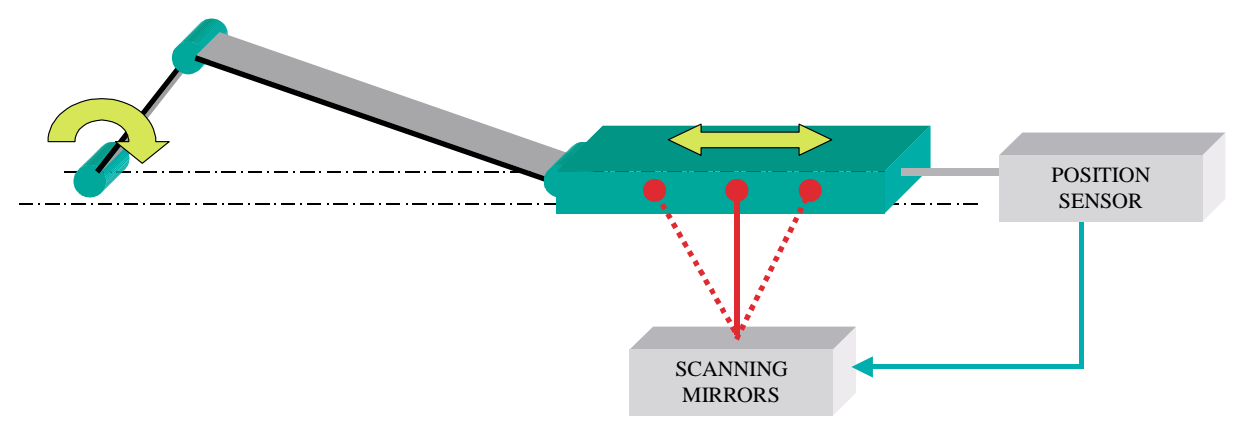

Fig. 11. Tracking of linear motion with linear position sensor in a mechanism.

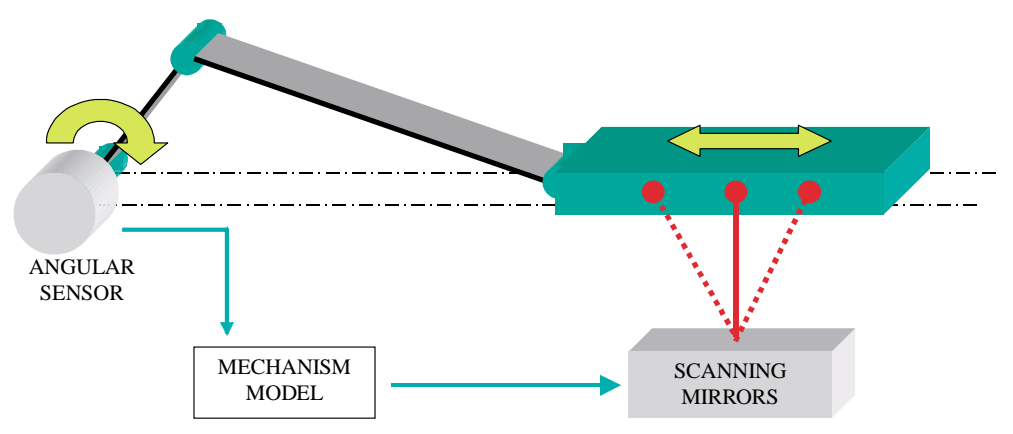

Fig. 12. Tracking of linear motion with angular sensor in a mechanism.

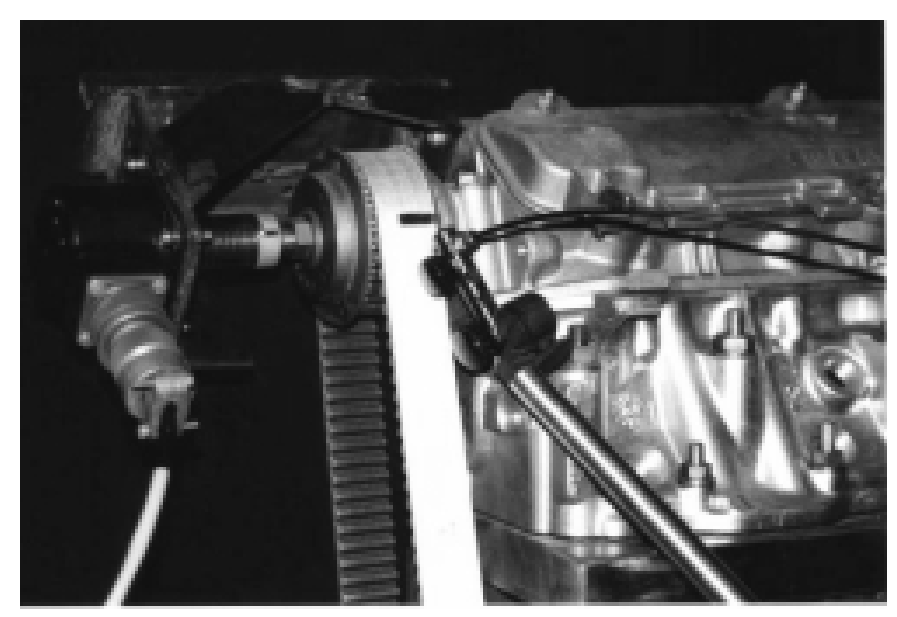

Fig. 13. The encoder and the trigger sensor for timing belt tracking.

The second effect is shown in Fig. 15.

The typical scheme of SLDV consider a couple of mirrors that move the measurement beam in a polar system, and therefore changing his angle respect the measured surface. In this way the angle between the laser beam (which is also the sensitivity direction) and the motion velocity is far from $90^{\circ}$ in quite every point. On the other hand a parallel scanning is quite difficult and to guarantee the perfect orthogonality is practically impossible.

For these reason traditional LDV measure a significant component of the tangential velocity VT, both in the DC and in the AC coupling, and this represent a disturbing input really difficult to be distinguished (Fig. 15(a)).

If the tracking approach is applied (Fig. 15(b)), the relative velocity between the belt and the beam, and therefore the disturbing velocity, become virtually zero 

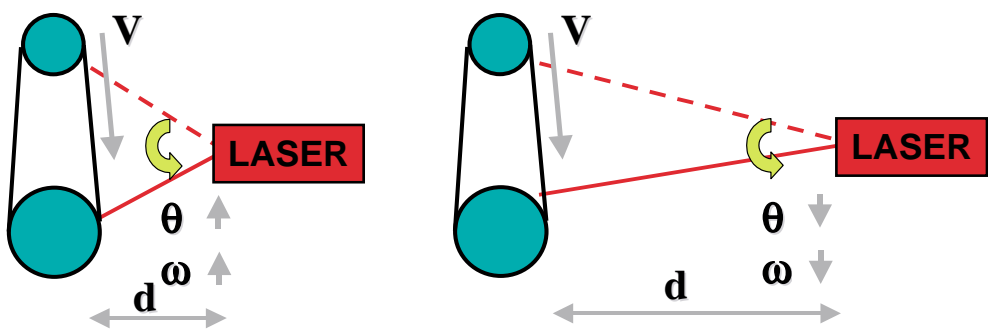

Fig. 14. Effect of vibrometer position on measurement parameters.
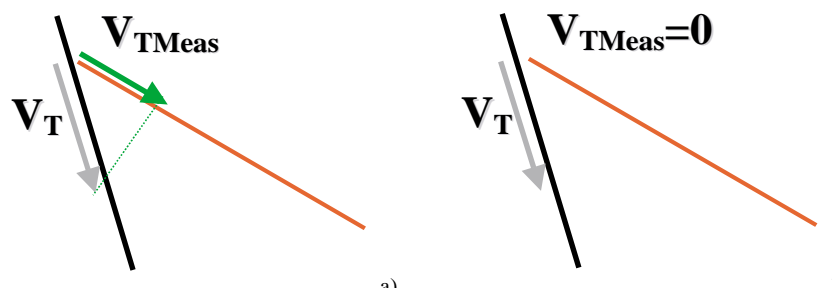

Fig. 15. Disturbing inputs without (a) and with the tracking (b).

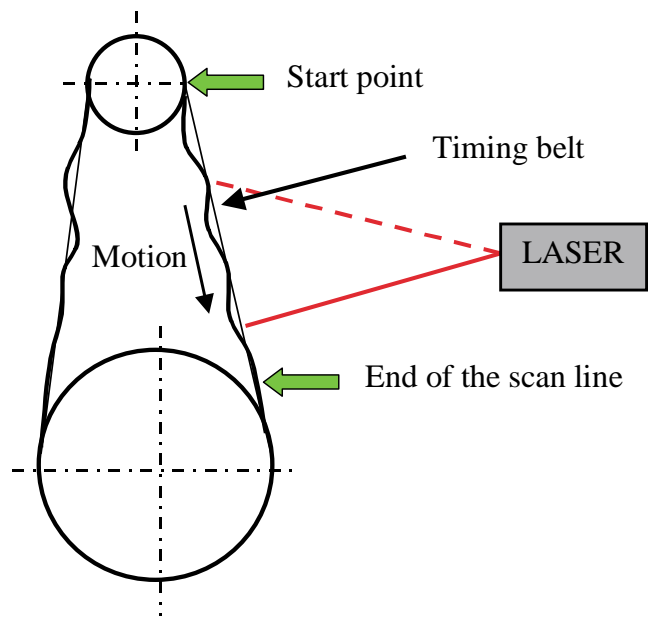

Fig. 16. Scheme of the tracking geometry.

or, at least, really low.

The TLDV is aligned in front of the belt, so that it can measure on one arm of it, between the two pulleys.

An optical sensor observing the belt cord provides the trigger signal. In fact when a retro-reflective stripe, drawn on the belt, is seen by the sensor the pulse signal give the information of the belt position and it can be used for the tracking trigger, so that each acquisition starts at the same belt (and not pulley) position.

Figure 16 describes schematically such an arrangement. Figure 17 shows the "grid" of points measured on the belt: due to the linear motion of the belt the complete $2 \mathrm{D}$ information of the vibration is provided

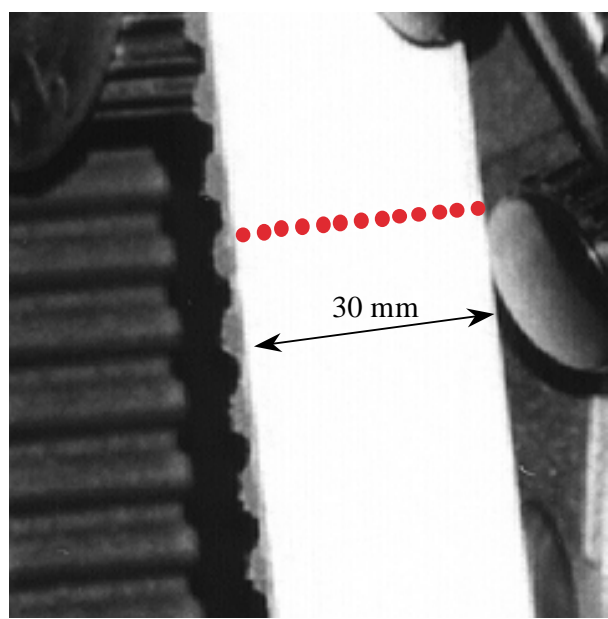

Fig. 17. The measurement grid across the belt.

by this simple grid, being the second coordinate represented by samples acquired in different position during the tracking.

The belt is scanned across a set of 13 parallel lines, each one originating from one of the grid points. The analogue velocity signal from the LDV is sampled during each scan; sampling rate can be fixed by an internal clock or it can be based on encoder output, so that time based or angle based data can be provided.

\subsection{The test bench for belt measurements}

A test bench has been designed that allows driving an automotive timing belt. Some pulleys drive the 


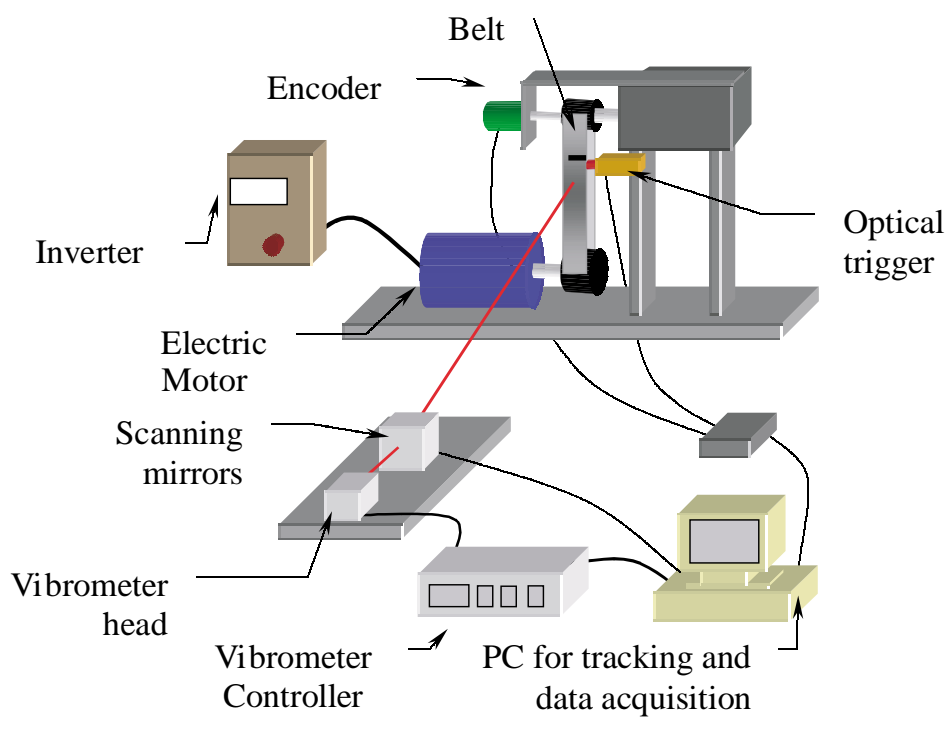

Fig. 18. Scheme of the TLDV measurement set-up.

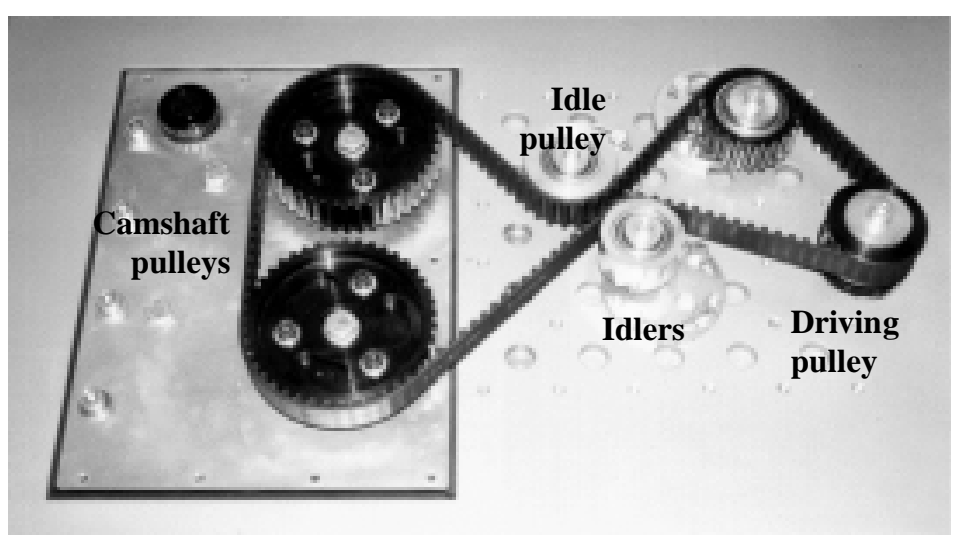

Fig. 19. The test bench.

belt; one is connected to an electric motor, another is connected to the camshaft of a four cylinder engine head. Figure 18 shows the scheme of the bench, while Fig. 19 shows a picture. An inverter can vary the speed of the electric motor, so that measurements at different camshaft speed can be done. The camshaft drives the four intake valves and the four discharge valves of the engine.

\subsection{Results}

The TLDV is aligned in front of the belt, so that it can measure on one arm of it, between two pulleys.

In Fig. 20 an example of results obtained on a timing belt at two different pulley speed, is reported. Vibration velocity is plotted versus time; it should be noted that for TLDV, if the laser beam moves at velocity VL and tracks the target point, then the time coordinate $t$ can also be considered as a spatial coordinate $x$. In fact the two are related to each other by the simple relationship of the space-time ratio:

$$
x=V_{L} t
$$

This also means that data are collected in the timespace domain, due to the motion of the measuring beam. This is typical of a Lagrangian type of observation. The signals in Fig. 20 therefore show what a moving observer measures on the belt when going from one pulley to the other.

It appears as an amplitude-modulated vibration signal. Its frequency depends on the excitation, mainly the meshing frequency of the belt teeth on the pulley, 


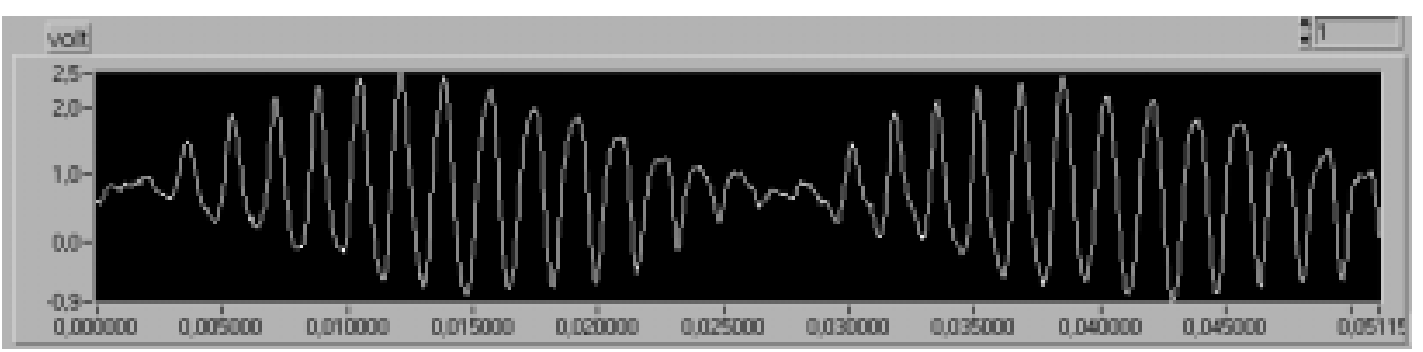

(a)

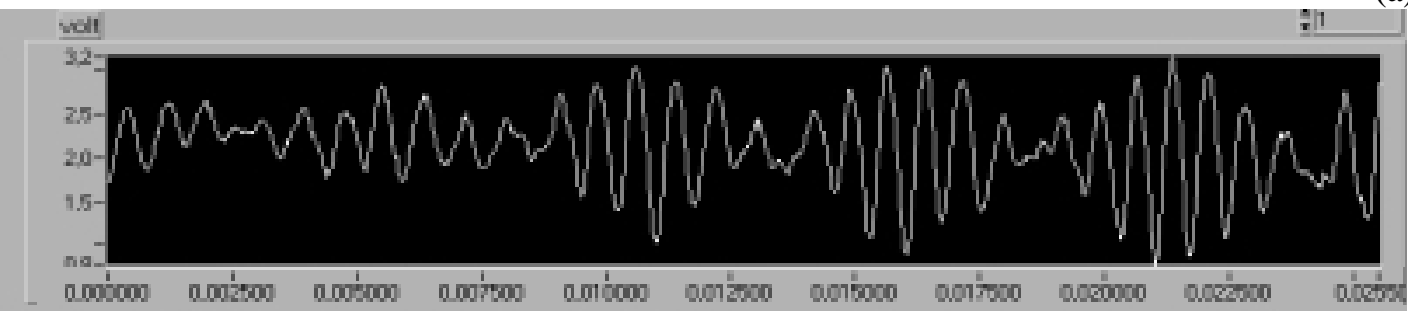

(b)

Fig. 20. Time histories of vibration velocity of a point of the grid at $\omega_{P}=335 \mathrm{rpm}$ (a) and $\omega_{P}=770 \mathrm{rpm}(\mathrm{b})$.

and on the belt dynamics. It is observed the belt vibration at this meshing frequency $\omega_{M}$, modulated by the operational deflection shape (in terms of velocity amplitude).

All recorded signal do not have a zero mean, as they should. This is a side effect of tracking, which is actually adding a non-zero mean velocity superimposed to real vibration velocity due to non-orthogonality between laser beam and belt. This produces low frequency components on all signals, which should be filtered out.

If belt speed changes then a different belt mode is excited and again we see vibration at the meshing frequency modulated by a different operational deflection shape, with a different number of nodes.

Measurements have been taken with a belt velocity up to $4.8 \mathrm{~m} / \mathrm{s}$.

The number of cycles of oscillation observed along one belt arm between two pulleys is constant. In fact, excitation frequency depends on number of teeth $z$ of each pulley and on its angular speed $\omega_{P}$, being

$$
\omega_{M} \propto\left(\omega_{P} z\right)
$$

If $T$ is the time needed to track the whole belt arm of length $L$, and then to record the vibration, we have

$$
T=\frac{L}{\omega_{P} R}
$$

where $R$ is the pulley radius, so that $\left(\omega_{P} R\right)$ is the belt velocity. This implies that the number of oscillations $N_{C}$ of the belt vibrating at frequency $\omega_{M}=\left(2 \omega f_{M}\right)$ for a time $T$ is equal to

$$
N_{C}=T \cdot f_{M} \propto \frac{L}{\omega_{P} R} \cdot \omega_{P} z \propto \frac{z L}{R}
$$

which is constant.

The operational deflection shape amplitude can be determined by taking the envelope of the measured signal along one belt arm. Nodal points are evident.

Figure 21 show typical power spectra computed from the data recorded by TLDV tracking a belt point at different belt speed.

Frequency analysis of the signals allows to observe the meshing frequency and its harmonics.

In Fig. 22 the front panel of the acquisition program is shown. In particular it is possible to note the average spectrum of every points of the grid.

In addition, choosing in such a spectrum a frequency, it is possible to plot the map of amplitude at that frequency on each point of the grid, which in this case is linear.

The data acquired on each point of the grid provide another vibration map on the time domain, which can be plotted. Figure 23 shows such a map at two different belt speed, built by plotting 13 parallel scans. Some phase errors are evident between parallel scans; this uncertainty is related to the triggering of the acquisition due to reflective strip oscillation. A larger peak-peak velocity is observed in the left part of the belt; this is probably related to geometric misalignment between the axes of the two pulleys that cause some difference in belt tension across its width. 

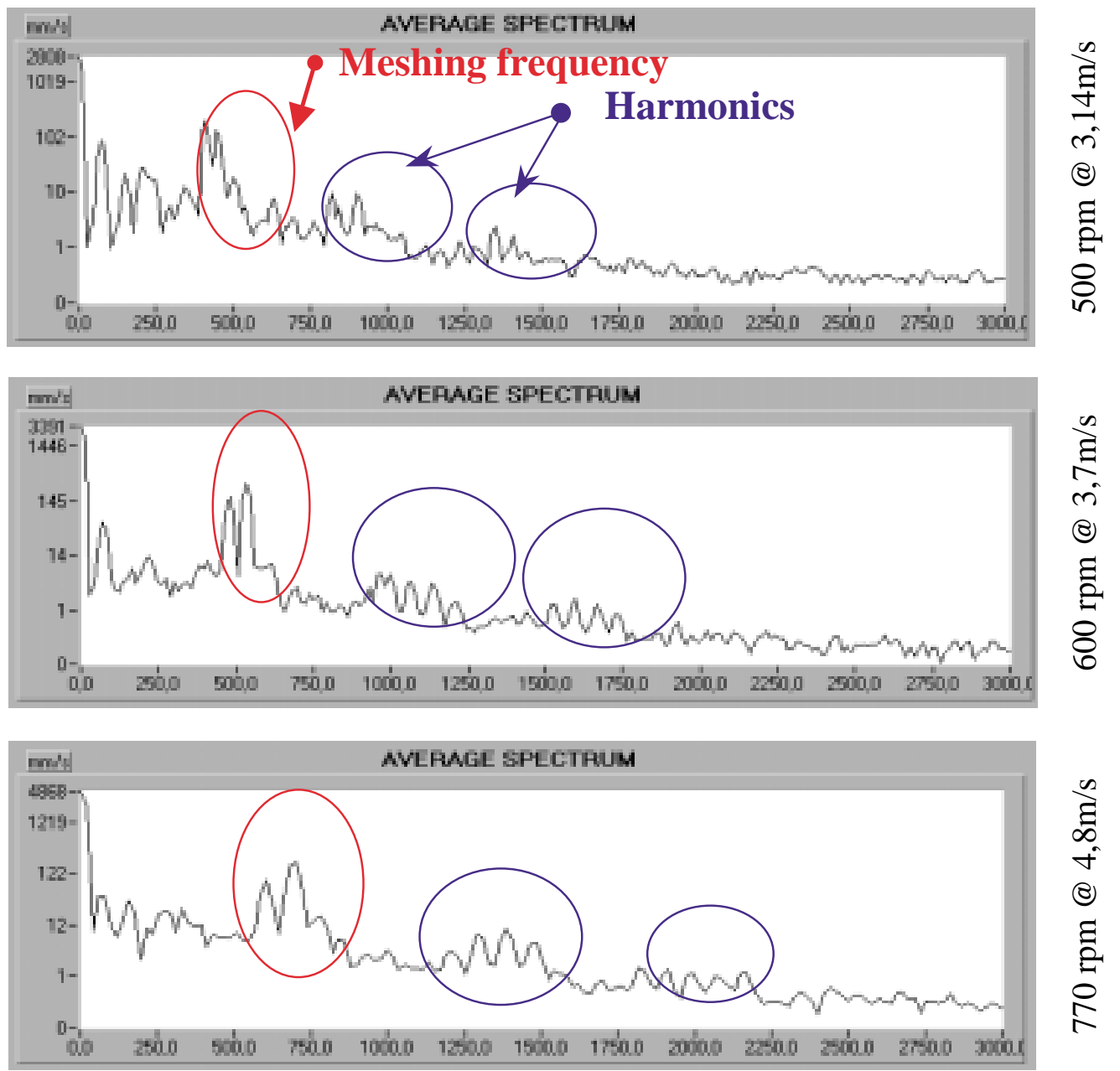

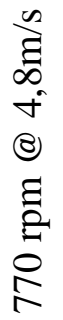

Fig. 21. Power spectral density of the measured vibration at different speed conditions.

\section{Measurement on windscreen wipers}

An advanced version of the TLDV was developed for specific applications adding the possibility to track arbitrary motions, as required by the application on complex motion mechanism.

In particular the system is now able to work with a-priori unknown motions through the application of a couple of sensors able to "feel" the actual position of the target point.

The Tracking Laser Doppler Vibrometer can be applied both for in-plane and out-of-plane vibrations of windscreen wipers in operative conditions.

The system is able to measure both on the wiper structure and on the rubber.

The measurement of the vibration of wipers in operative conditions is a very complex task.
In fact, they are very light and they have low stiffness and small structures; the application of contact sensors, such as accelerometers, is not possible. The case of the assessment of vibration of the rubber of the wiper is a very convincing example.

In some cases the mechanism model is not available, or it is really complicated and difficult to be used. In such cases the application of the same approach shown for timing belt application appears not convenient. An example of this is the case of some wipers. In fact, if for traditional systems on simple windscreen the motion is basically circular and planar, in some cases the problem become more complex and some sensors, directly linked to the moving mechanism, must be applied. The moving structure position signal can be obtained using various kinds of transducers, depending on the moving structure geometry and on the measure type (in-plane or out-of-plane vibration). 


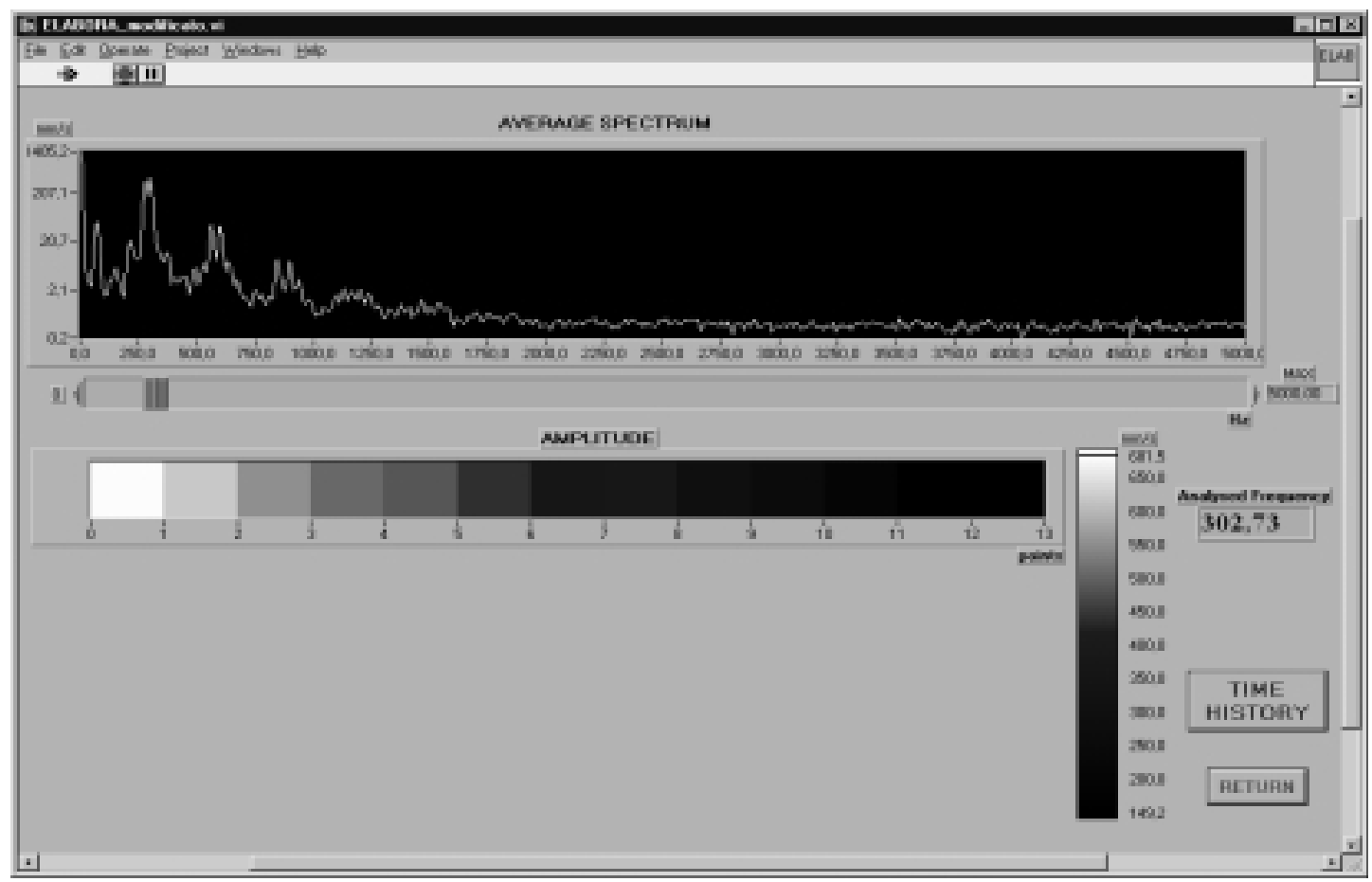

Fig. 22. Presentation panel of the tracking software: average spectrum and amplitude map at a selected frequency.

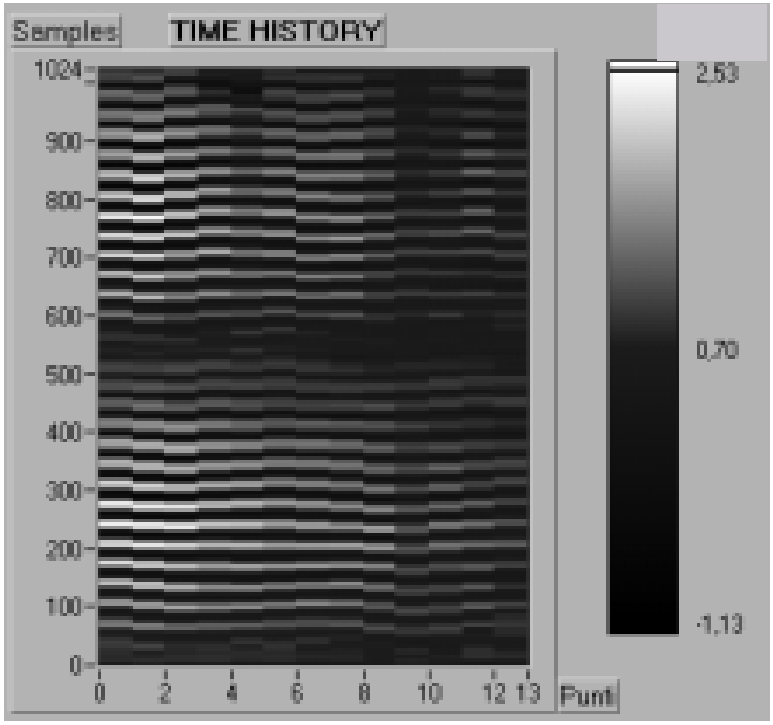

(a)

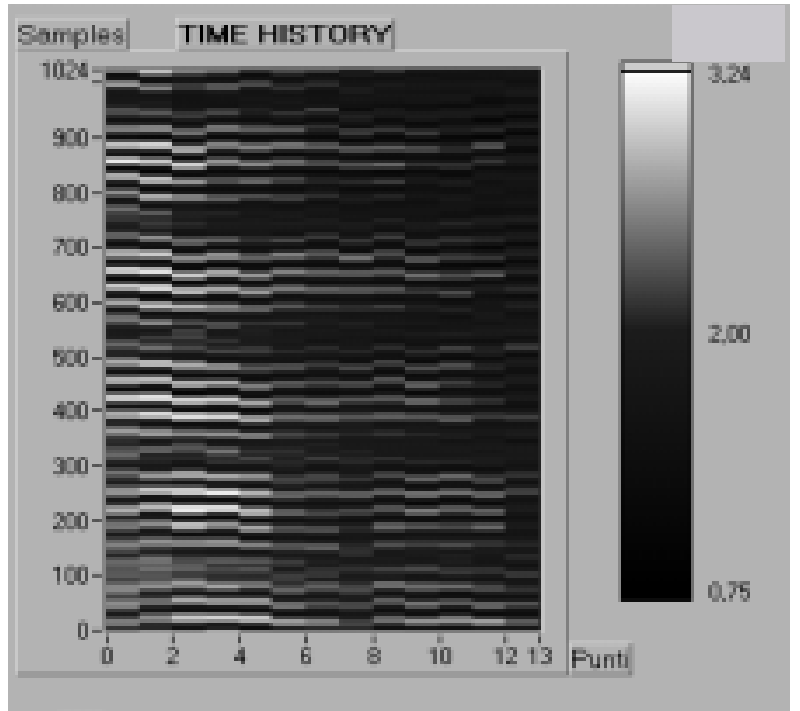

(b)

Fig. 23. Time-space map of timing belt vibration velocity ( $y$ direction) of different points across the grid ( $x$ direction); (a) at $u p=335$ rpm and (b) $\omega_{P}=770 \mathrm{rpm}$.

Therefore, for in-plane vibration measurements on a simple wiper mechanism were performed using a standard incremental rotational encoder as position transducer. The tracking algorithm is based on analytic re- lationship depending on the geometry of the system, as shown in Fig. 24. In particular, after the evaluation of the relative position between laser vibrometer and the wiper, the system compute the trajectory for each en- 


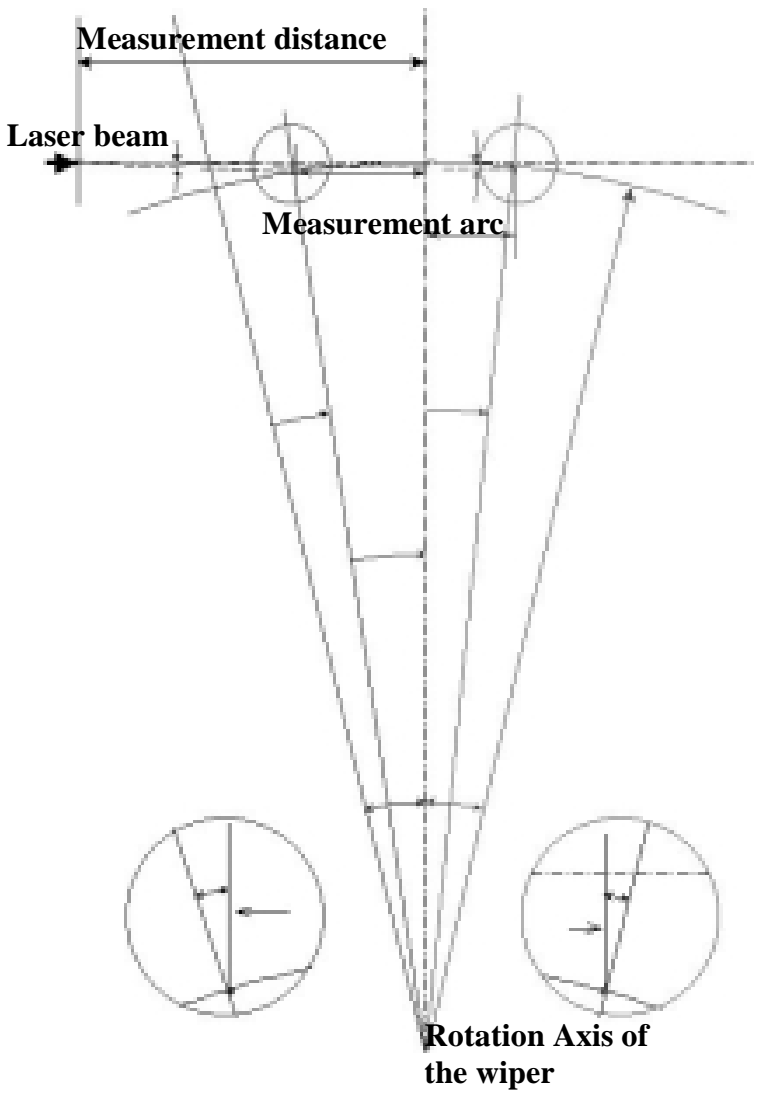

Fig. 24. Geometrical element of the system used for the in-plane vibration measurement.

coder pulse ( 0.5 degree) starting from the trigger point. Due to the fact that the target point's trajectory is apriori known, D/A buffered generation was used, as already discussed for the tracking of the belt. For outof-plane measurements on a complex motion wiper a couple of wire potentiometer was applied.

Potentiometer output, proportional to the distance of the target point (connected to the end of the wire) from the references points (the constraints of potentiometers) is acquired using an A/D board, which resides in a PC.

Subsequent to elaboration by proper software these signals will represent the actual position of the target point.

In fact, being the trajectory a-priori unknown, it is not possible to use the buffered A/D strategy.

Of course, some knowledge of sensor characteristics and geometrical arrangement of the rig are in any case necessary in order to translate sensor signal in the information of target position.

The geometrical scheme of the motion plane of the out-of-plane measurement system is shown in Figs 25 and 26.

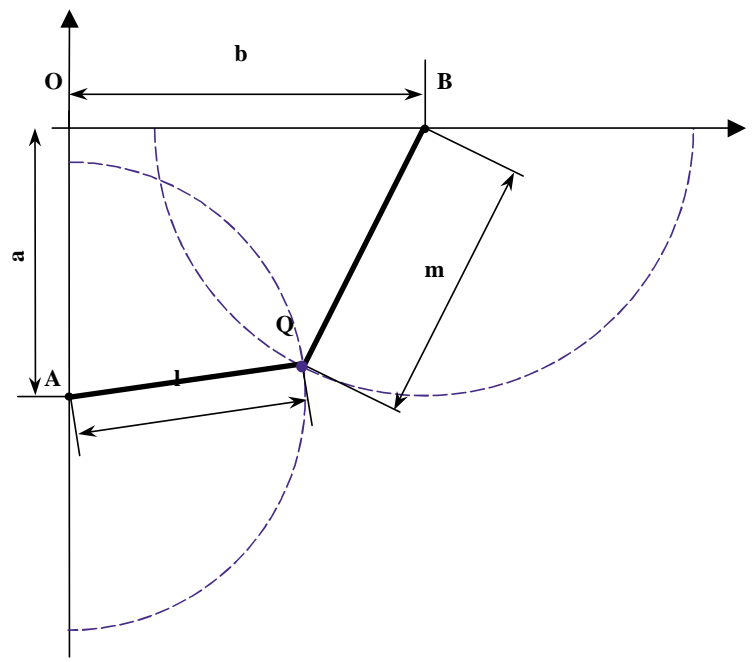

Fig. 25. Geometrical scheme of the motion plane for the out-of-plane vibration measurement.

In the set-up phase the program gets some system physical parameters, needed by the tracking algorithm.

In the tracking phase, potentiometers instantaneous output are acquired and properly processed, using information from the set-up phase and calculating the point $\mathrm{Q}$ which is one of two intersection points of two circumferences defined by the wires length (radiuses) and potentiometer locations in the Cartesian co-ordinate system (coordinates a and $b$ ). One intersection point is rejected by a simple sign condition, thus the target point $\mathrm{Q}$ is univocally defined.

Such calculation, that must be performed while the object moves, is the "bottle-neck" of the system. Performances are therefore depending on computation capabilities of the used PC.

Due to the fact that no predefined trajectory was used, because in the arbitrarily motion trajectory are unknown, in this application was not possible to use D/A board buffered signal generation feature. The only way to increase system performances is the optimization of the tracking algorithm.

\subsection{The test benches for windscreen wiper measurements}

Different kind of tests was performed both on laboratory simplified benches with a flat screen, but well representing the real operating conditions.

The bench arrangements allow to consider both rotational motion wiper and complex motion wiper, as shown respectively in Figs 27 and 28. The windscreen wipers are mounted on a rigid metal structure and works 


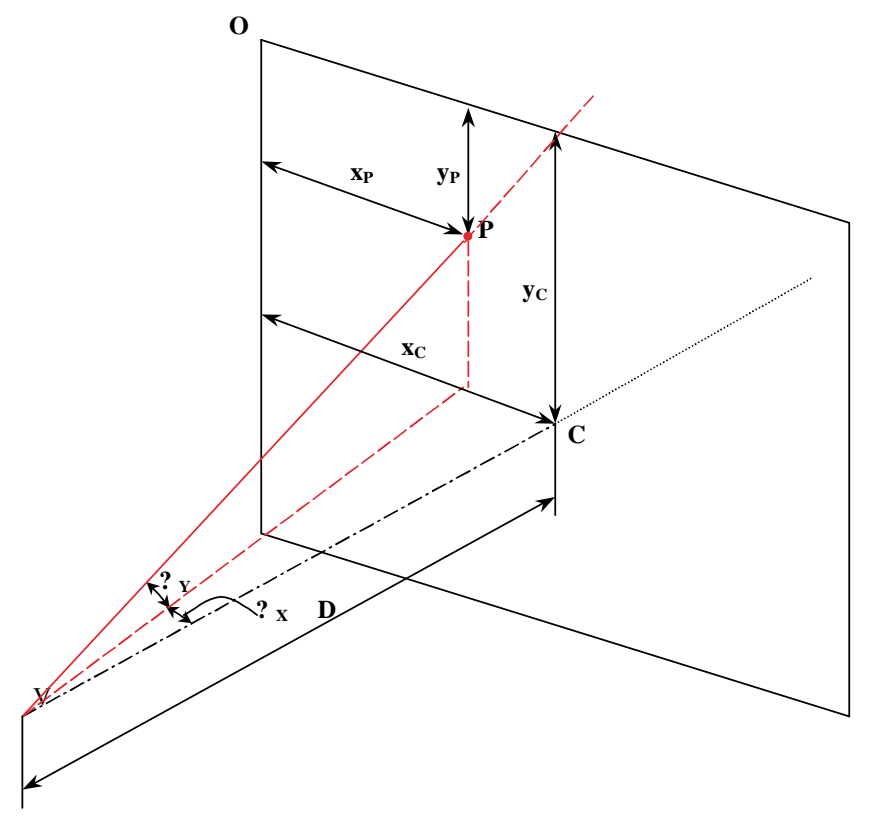

Fig. 26. Measurement system geometry.

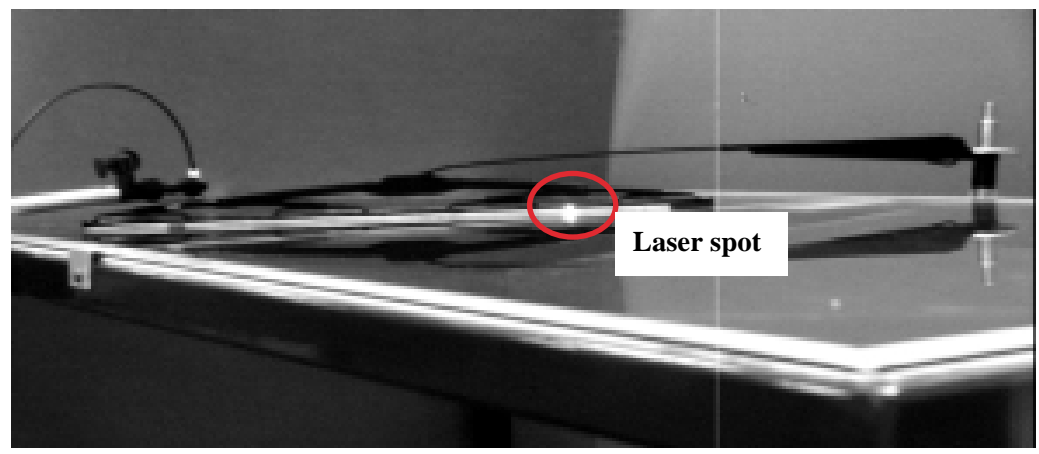

Fig. 27. Simple windscreen wiper bench for in-plane and out-of-plane vibration measurement.

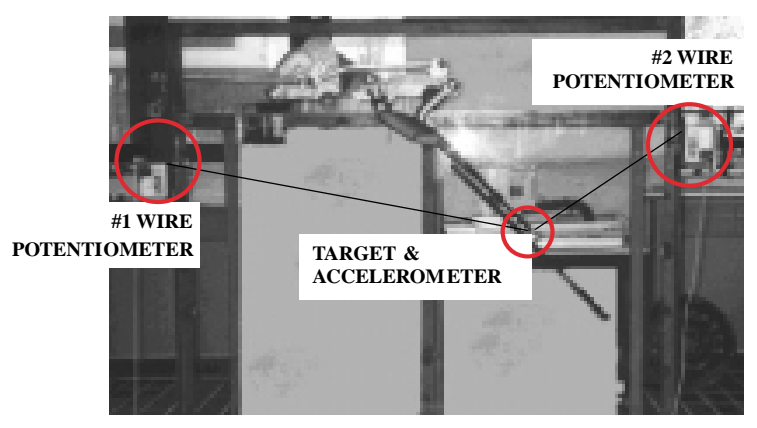

Fig. 28. Complex windscreen wiper bench for out-of-plane vibration measurement.

on a flat transparent glass. Wire potentiometer and trigger are visible. The standard Polytec laser-scanning head was used.

The trigger signal is provided by a fiber optic proximity sensor, which operates through the glass and is sensitive to wiper passage. Different colors of the sides of the wiper rubber guarantee the trigger starts always in one wiper motion direction.

References can be obtained in different ways. In particular an idea of the vibration characteristics of the rubber blade can be provided by an accelerometer mounted on the glass screen.

On the other hand, some tests are performed in order to verify the tracking performances of the system. In these cases, an accelerometer was mounted on the wiper structure, and the tracking is performed on the accelerometer surface (the target point). 


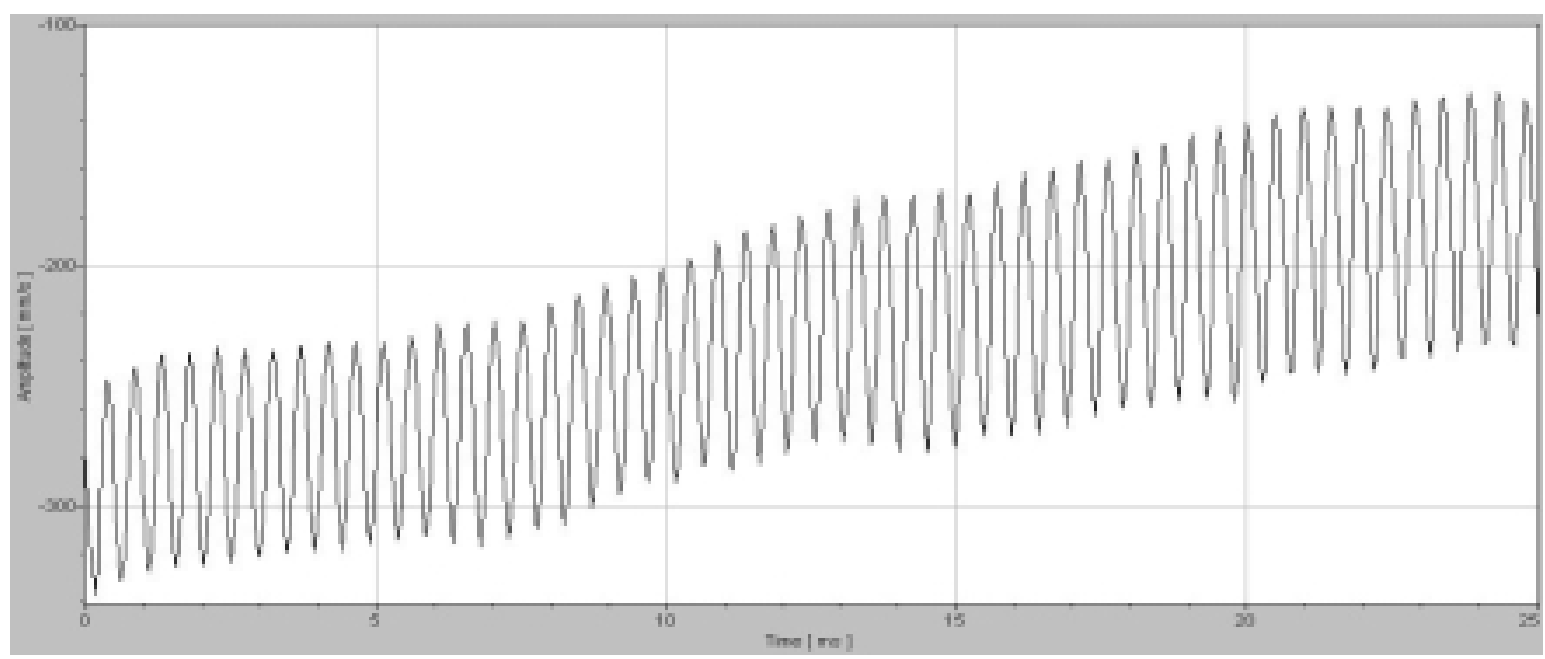

Fig. 29. Time history of in-plane vibration during tracking.

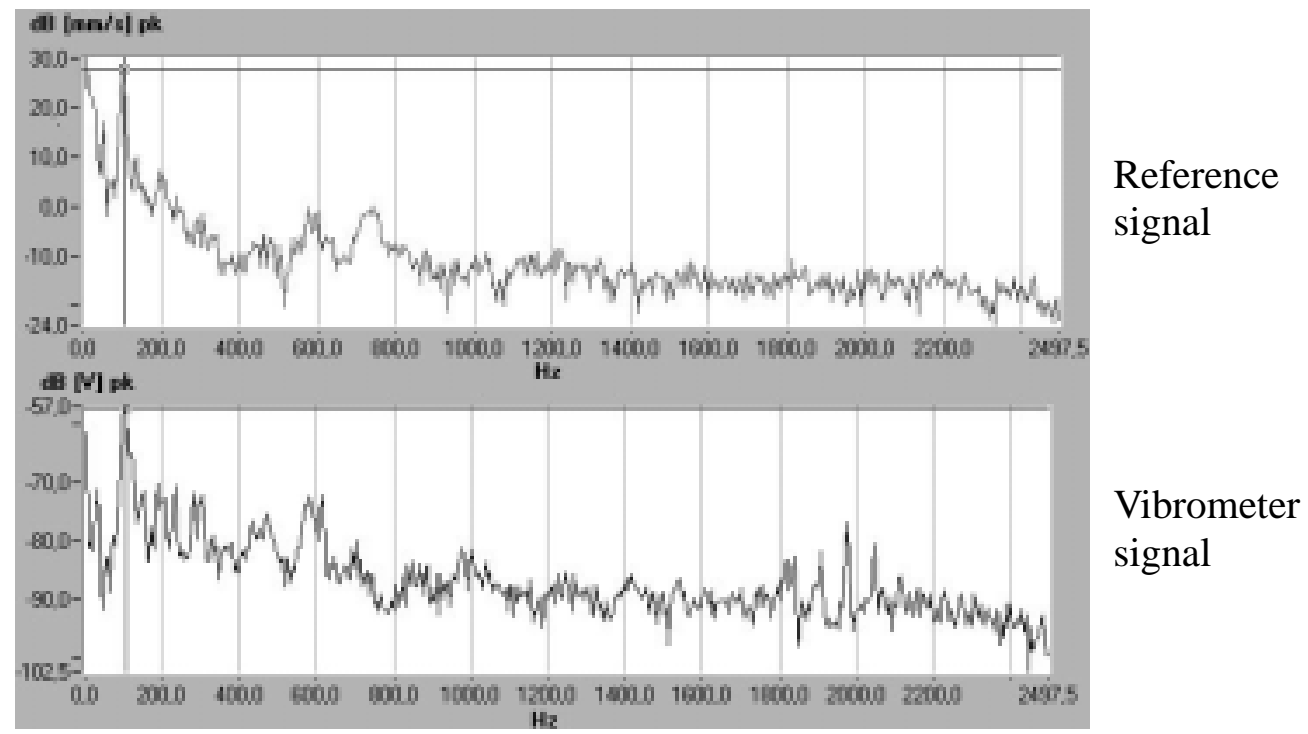

Fig. 30. Spectra of signals from vibrometer and reference accelerometer on the windscreen.

The in-plane and out-of-plane vibrations are analyzed, depending on test rig arrangement and tracking strategy chosen.

\subsection{Results}

In order to completely evaluate the vibration behaviour of a windscreen wiper and to check performances and versatility of the Tracking Laser Doppler Vibrometer, both in-plane and out-of-plane vibration was measured.

In particular two types of wipers with different motion were tested: a wiper with typical rotational motion and a wiper with more complex motion (translational motion added to a rotational motion). For the out-ofplane vibration measurement both the wipers motion trajectories was considered unknown, in order to test the tracking strategy based on wire potentiometers.

\subsection{Measurement of in-plane vibrations}

In Fig. 29 the time history of in-plane vibration during tracking is shown.

In Fig. 30 a comparison between spectra obtained from vibrometer and reference signal is shown. In this 


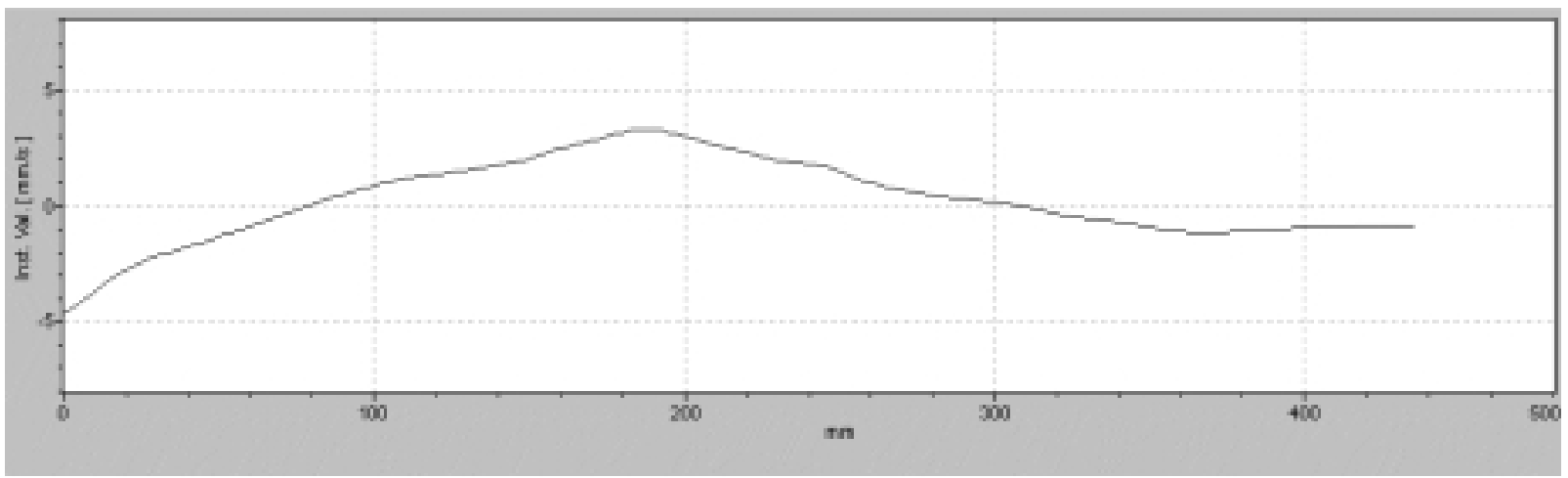

Fig. 31. Operational Mode Shape of the rubber blade at $123 \mathrm{~Hz}$.

case the reference is obtained with an accelerometer attached on the windscreen (is impossible to attach it on the rubber blade). These two signals are not exactly representing the same vibration point, but offer a good idea of the phenomenon. In fact, the glass and the rubber are in direct contact and represent the opposite face of the slide, generating vibrations.

As is possible to note, a good agreement is present between two spectra, with the same vibration signature in particular in the frequency range $(50-300 \mathrm{~Hz})$ in which the acoustic noise is present. Of course the different characteristics of the structure determine a different weighting of the peaks in the spectra.

In Fig. 8 the Operational Mode Shape of the rubber blade at $123 \mathrm{~Hz}$ is shown.

Multiple measurements along a line over the rubber blade make possible an analysis similar to that of a traditional Scanning Vibrometer.

Tracking of each point is not obtained simply progressively increasing the mirror deflection, but requires geometrical model adaptation with subsequent specific calculations. In fact, each point of the grid is characterised by a different trajectory, which must be calculated, point-by-point, before the scanning operation.

It is important to note that the frequency resolution of the analysis is of course determined by the acquisition/tracking time, i.e. is depending by the wiper velocity and the arc amplitude and it is not an independent variable.

\subsection{Measurement of out-of-plane vibrations}

\subsubsection{Rotational motion windscreen wiper}

The simple system geometry (Fig. 27) allows windscreen wiper tracking using only an encoder or one potentiometer (solution used in this test).
The vibrometer is in front of the glass, observing vibration of the wiper arm orthogonal to the windscreen; no external excitation was used.

In order to verify the tracking performances in this case is possible to put an accelerometer on the target point of the arm. Of course such solution is not possible when the interest is on the vibration of the wiper for the mass load induced by the accelerometer, and this solution was used only for a metrological analysis of TLDV system.

To verify the capability of the system to work following "industrial" requirements, no accurate (and slow) alignment was performed between the rotational axis of the wiper and scanning axis of the vibrometer.

Such geometrical configuration determines a large variation of the optical path of the laser beam during the tracking of the wiper running on an arc trajectory.

Such noise is evident in the vibrometer signal with a pedestal (Fig. 32(a)) on which the vibration signal is superimposed. The signal this pedestal can easily be eliminated by filtering or digital interpolating.

The comparison between vibrometer and accelerometer signal shows a good agreement. Obviously the reference signal is not affected by the pedestal noise (Fig. 32(b)), but the correspondence between time histories, obtained with a synchronised sampling, is really good. The spectra (Figs 32(c) and (d)) allow to better appreciating the correspondence between signals.

\subsubsection{Complex motion windscreen wiper}

This bench differs from the previous one only for the cinematic scheme of the windscreen wiper (Fig. 28); its motion is the result of the addition of a rotational and a translational motion. Such difference suggests a completely new approach in the tracking strategy, being too much complicated the analysis of the trajectory of each point of the wiper arm. 


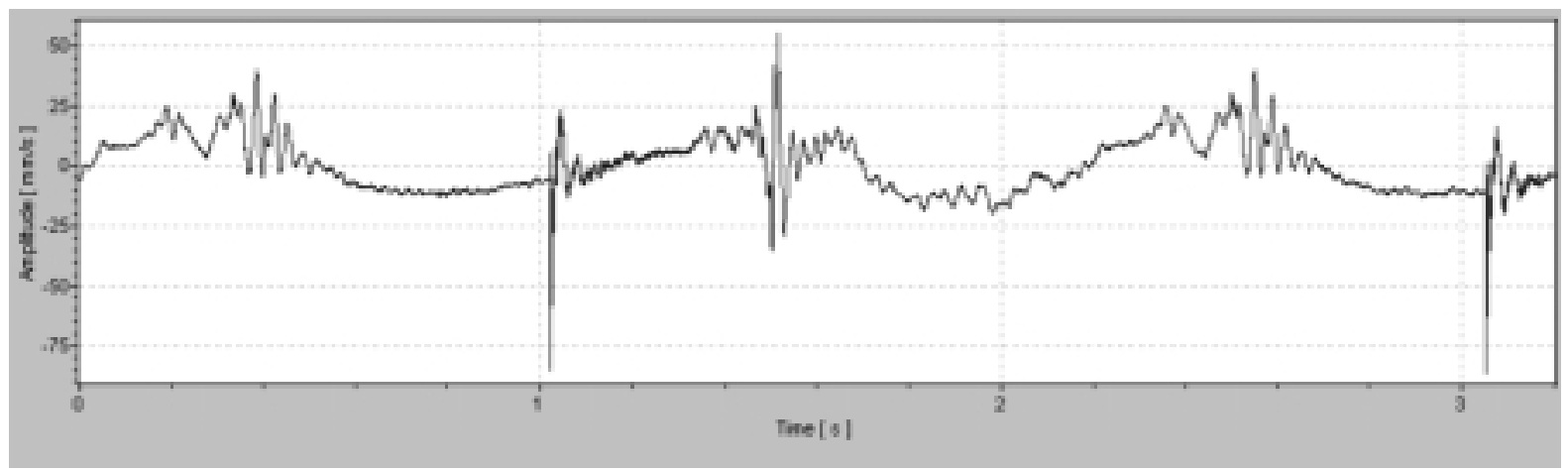

a) Vibrometer signal time history.

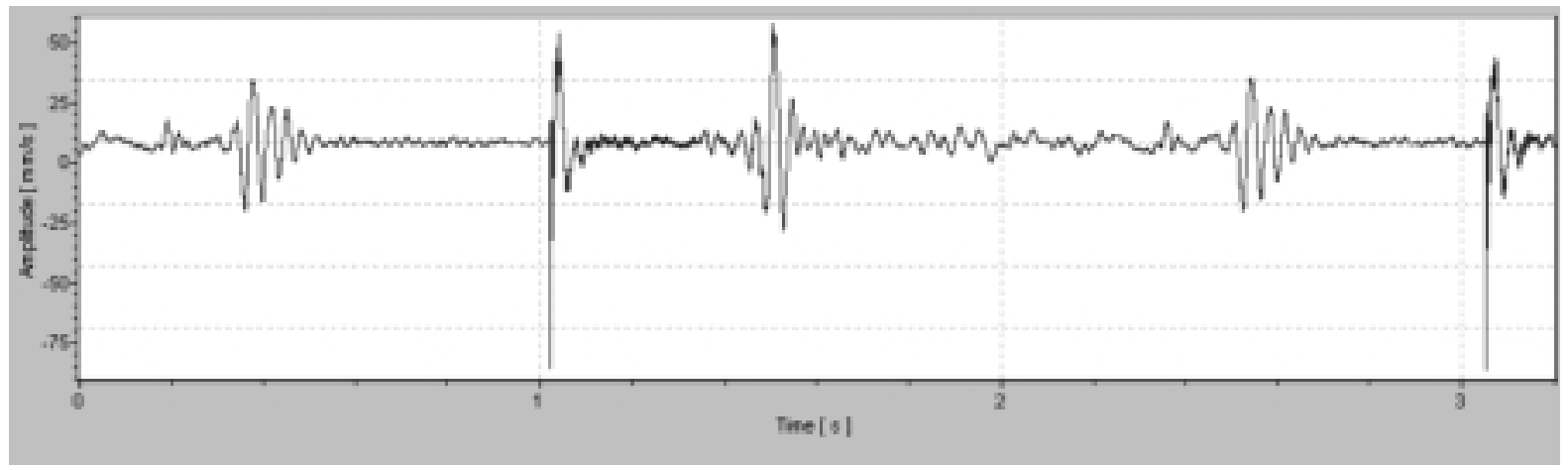

b) Accelerometer signal time history.

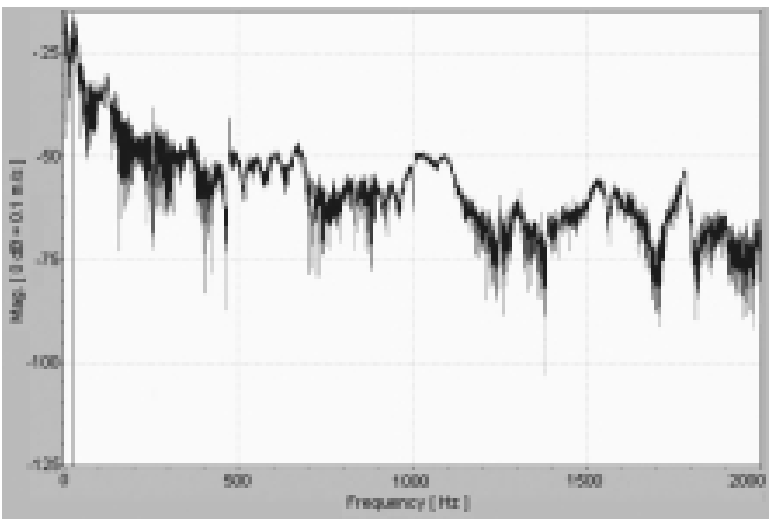

c) Vibrometer signal spectrum amplitude.

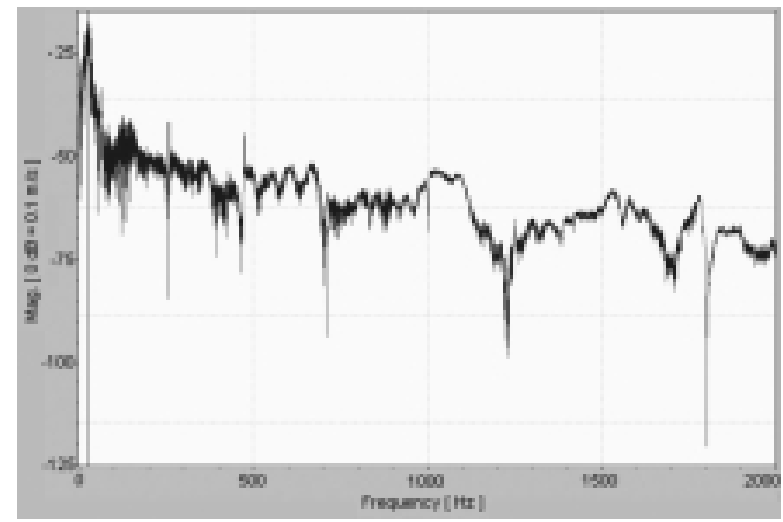

d) Accelerometer signal spectrum amplitude.

Fig. 32. Results on rotating windscreen wiper.

In Figs 33(a-d) vibrometer and accelerometer time history signal and amplitude spectrums, related to the measurement on the windscreen wiper with complex motion are shown. Spectrums are obtained with 30 averages. Vibrations were produced by windscreen wiper motion; no external excitation was used.

About the results there is a good correspondence be- tween the vibrometer signal and the accelerometer signal. As in the case of rotating windscreen, in the vibrometer time history is visible a very low frequency motion component, related to the laser path length variation during the tracking, superimposed to the vibration signal. This effect is also visible in the vibrometer spectrum. 


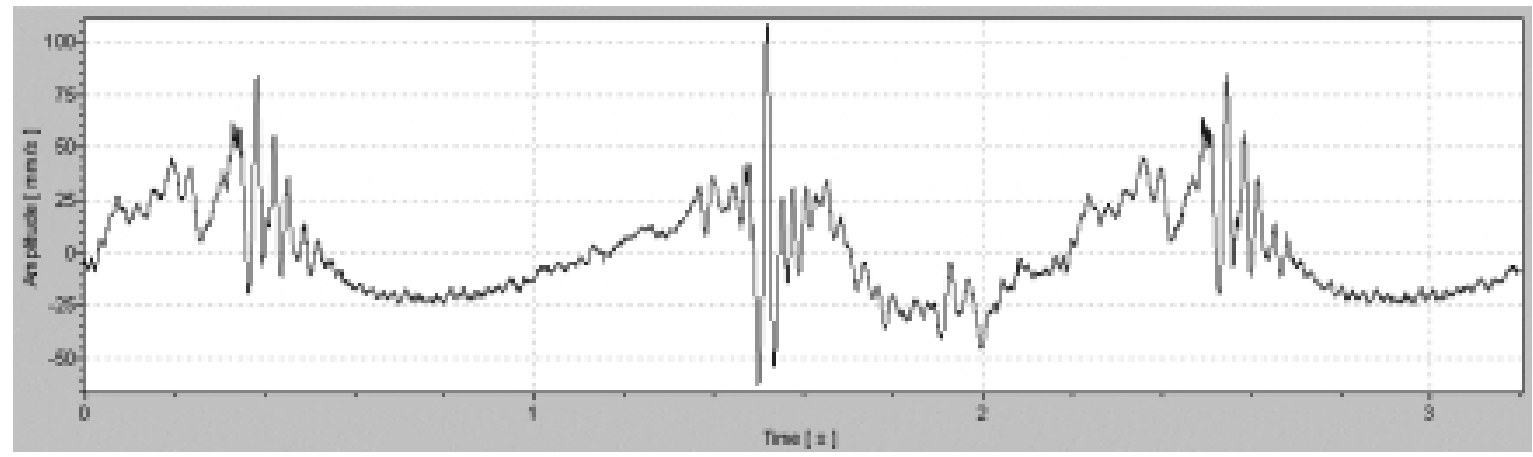

a) Vibrometer signal time history.

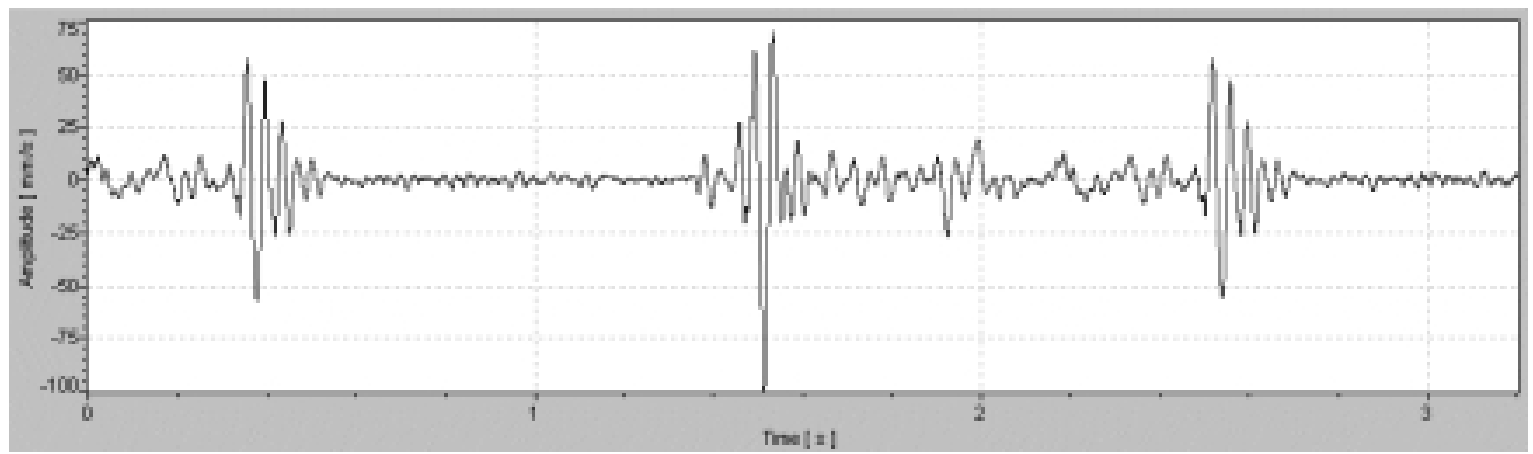

b) Accelerometer signal time history.

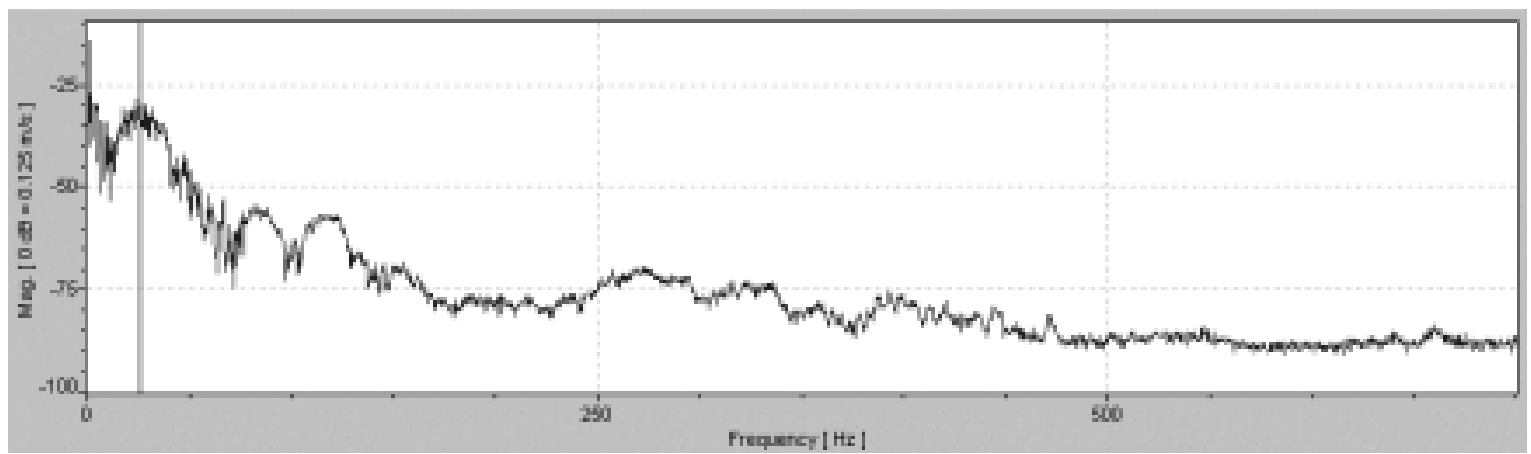

c) Vibrometer signal spectrum amplitude.

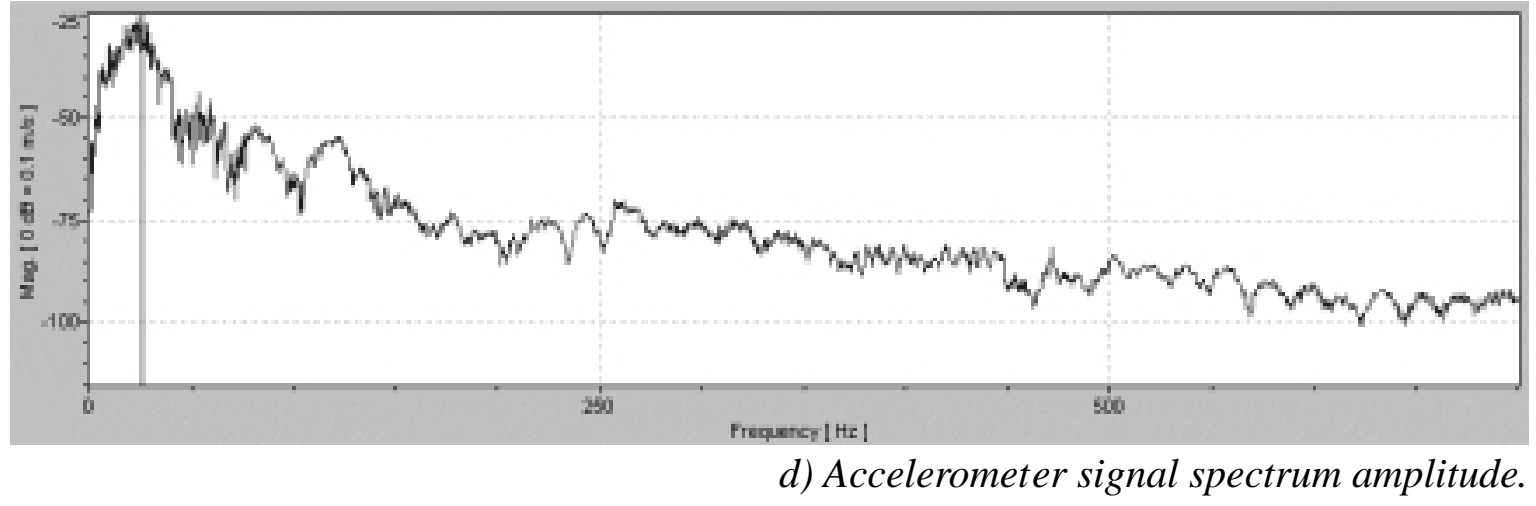

Fig. 33. Results on complex motion windscreen wiper. 


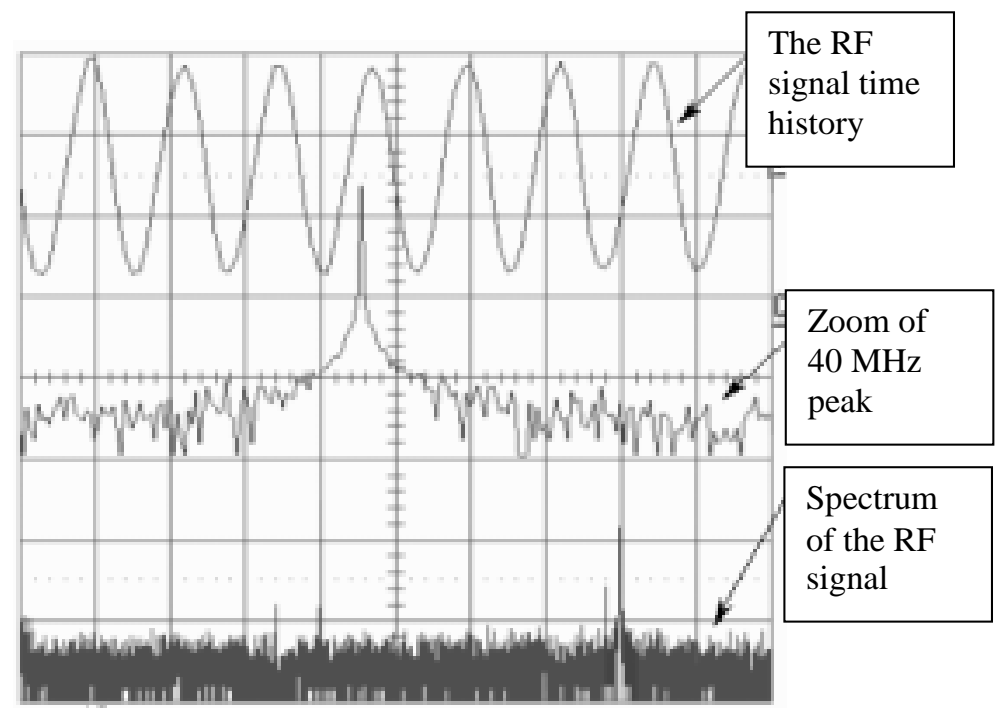

Fig. 34. RF signal with both target and laser beam steady.

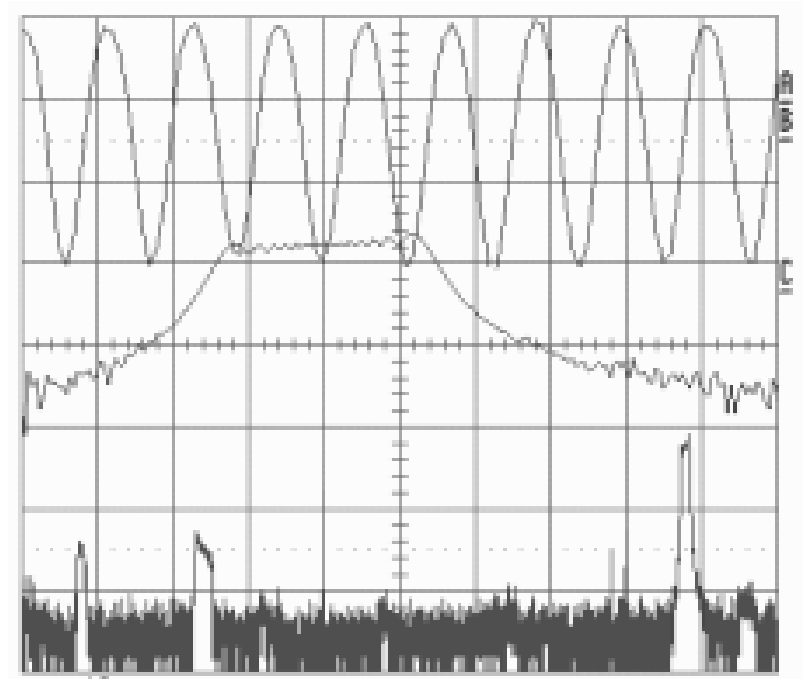

Fig. 35. RF signal with target moving and laser beam steady.

\section{Assessment of velocity accuracy through $\mathrm{rf}$ signal analysis}

In order to assess the effect of the tracking approach on the accuracy in the velocity measure an analysis of the Radio Frequency (RF) signal was performed.

In fact observing the RF signal is possible to extract a direct indication of the SNR on the measurement induced by the relative movement between the measuring beam and the target surface. The RF signal represents the direct image of the optical signal (and in particular interference and speckle noise), not yet affected by the tracking filter and by the demodulator.
As well known, the RF signal at the output from the interferometer is given by

$$
S_{R F}=A \cdot \sin \left\lfloor\left(\omega_{40 \mathrm{MHz}}+\omega_{\text {Doppler }}\right) \cdot t\right\rfloor
$$

The frequency carries the information of the vibration velocity, and this is what the demodulator must extract.

In order to assess the optical quality of the measure two different features of the RF signal must be considered:

1. the SNR in the sense of the ratio between the amplitude of the peak and the average height of the noise pedestal; 


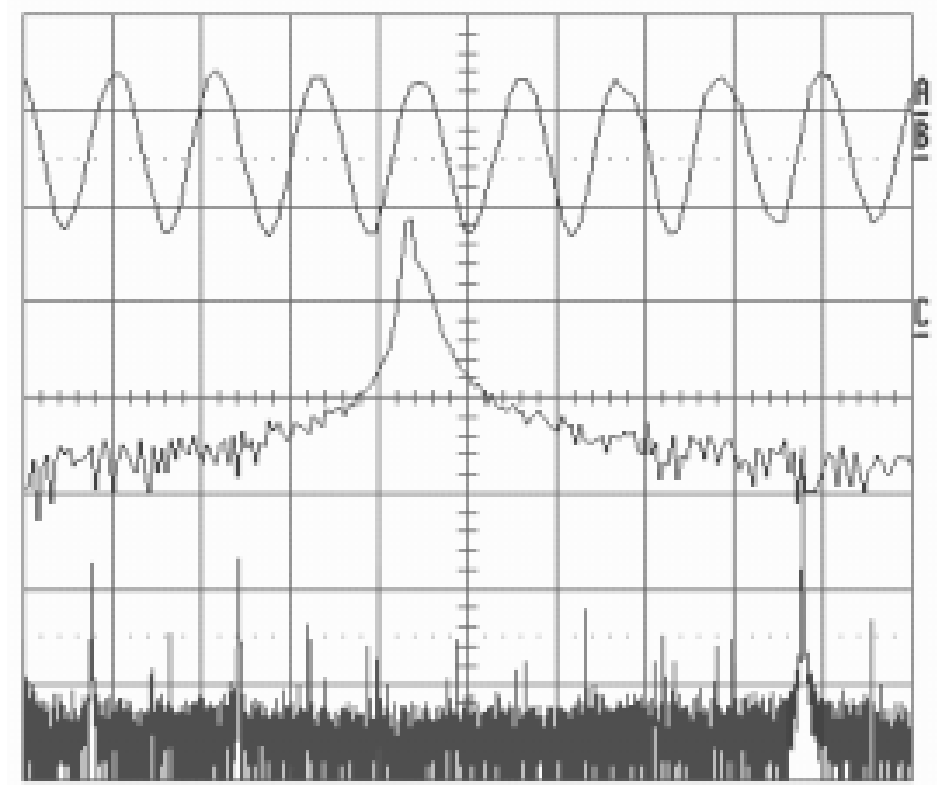

Fig. 36. RF signal with target moving and laser beam in tracking conditions.

2. the "shape" of the peak, in sense of the range of variability of the peak frequency not due to the change in the surface velocity.

In Fig. 34 the reference conditions, with both target and measurement beam steady on a retro-reflective tape, are reported. This signal represents the best possible for each specific interferometer.

In particular the RF signal and the corresponding spectrum are reported, with the zoomed details.

Without vibration the $40 \mathrm{MHz}$ carrier is very well defined $(\mathrm{SNR}=66 \mathrm{~dB})$.

In Fig. 35 an example of RF signal with moving object and measuring beam steady is reported.

The peak shows the typical shift due to the Doppler effect. The acquisition period, $10^{-4} \mathrm{~s}$, allows to consider negligible the effect of the variation of the velocity of the target.

It is evident the variation in the signal quality. The SNR is now about $44 \mathrm{~dB}$ due to energy distribution in a wider range and the peak shape determine a large frequency uncertainty (a range of about $0.6 \mathrm{MHz}$ ).

In the considered interferometer (with an $\mathrm{He}-\mathrm{Ne}$ laser) this frequency uncertainty corresponds to a velocity ambiguity of about $\pm 1 \mathrm{~ms}^{-1}$. This is due to noise effect (such as speckle noise) due to relative motion between the laser beam and the target.

In Fig. 36 an example of RF signal in tracking conditions, i.e. with moving object and measuring beam fixed on it, is reported.
The signal quality is improved. The SNR is now about $56 \mathrm{~dB}$ and the peak is thin. The noises are quite eliminated by the virtual absence of relative velocity between the target and the measuring beam.

The velocity uncertainty is significantly reduced.

\section{Conclusions}

This paper describes the recent developments of the Tracking Laser Doppler Vibrometer for the analysis of moving object in the automotive world.

In particular, the application to the analysis of vibration of tyres during his rotation in a drum test bench, of a timing belt in working conditions and of a windscreen wiper operating on the glass was presented.

The different versions of the system demonstrate its capability to measure the time histories of the velocity on the analysed component and therefore to evaluate the excited frequency and the damping effects.

Some examples of results and of signal processing are shown, together with a discussion on measurement problems.

\section{Acknowledgement}

The activity described in this paper was financially supported by the EC within the project $\mathrm{n}^{\circ} \mathrm{BRPR}-\mathrm{CT} 97$ 0594 VALSE. 
The author would like to acknowledge for the precious collaboration all the partners of this project and in particular the Bridgestone-Firestone Technical Centre Europe (Rome, Italy) in the persons of Dr. F. Baldoni and Dr. G. Ingenito, and the Robert Bosch GmbH (Stuttgart, Germany) in the persons of Dr. M. Fischer and Dr. K. Bendel.

\section{References}

[1] P. Castellini, G.M. Revel and E.P. Tomasini, Laser Doppler Vibrometry: a Review of Advances and Applications, The Shock and Vibration digest 30(6) (1998), 443-456.
[2] P. Castellini and C. Santolini, Vibration measurements on blades of naval propeller rotating in water with Tracking Laser Vibrometer, Measurement 24 (1998), 43-54.

[3] P. Castellini and N. Paone, Development of the tracking laser vibrometer: performance and uncertainty analysis, Review of Scientific Instruments 71(12) (December 2000), 207-215, ed. American institute of Physics, Woodbury, NY.

[4] P. Castellini and G.M. Revel, Tracking laser vibrometer measurements for estimation of excitation level in rotating structures, 17th International Conference on Modal Analysis, Orlando, 1999, pp. 1005-1011.

[5] P. Castellini and R. Montanini, Joint time-frequency analysis of tracking laser Doppler vibrometry data on a rolling tire, Sensor 2001 Conference, Numberg, June 2001.

[6] P. Castellini and R. Montanini, Vibration measurements on automotive rolling tires: a Lagrangian approach, ATA 2001 Conference, Ancona, September 2001, published on CD-ROM. 

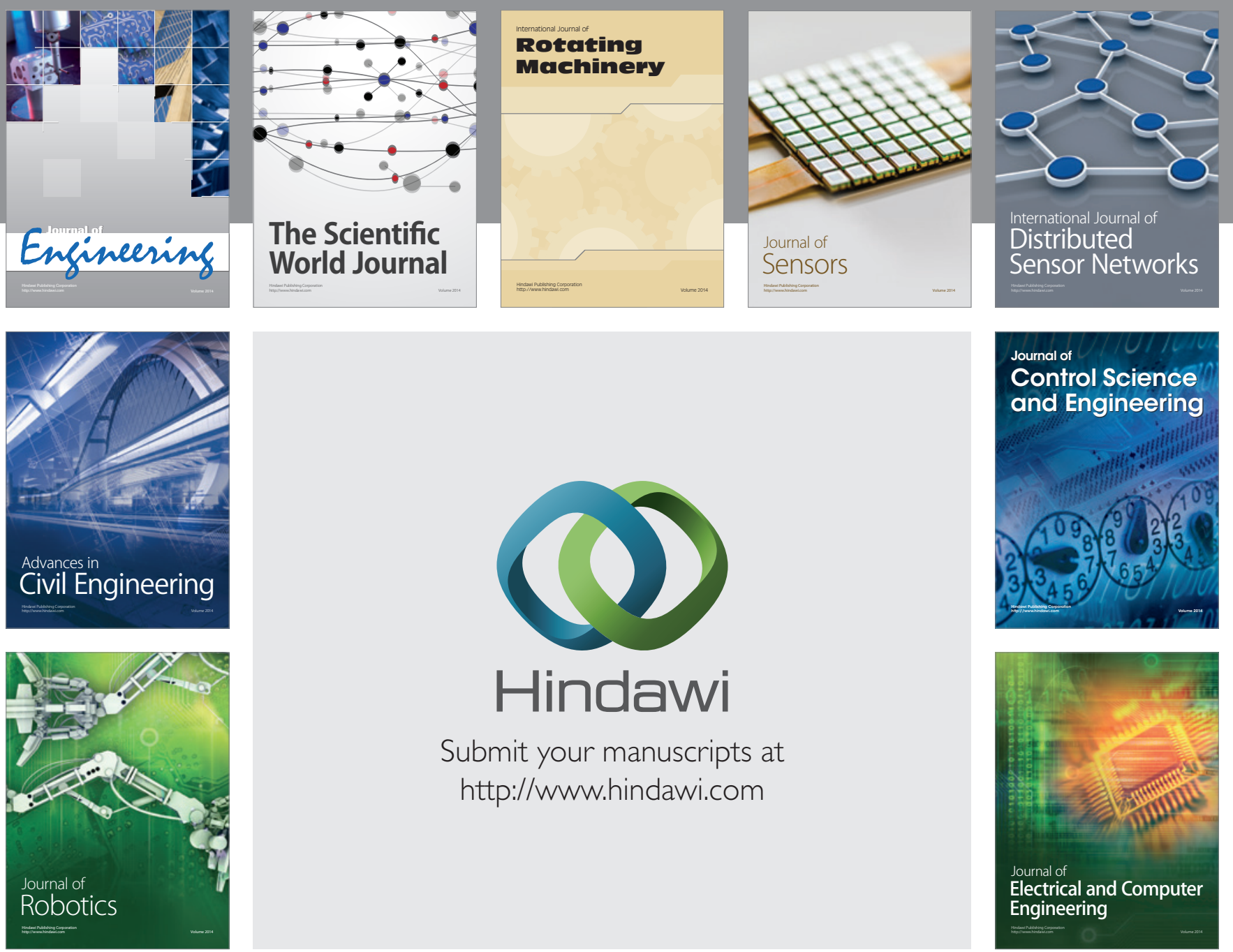

Submit your manuscripts at

http://www.hindawi.com
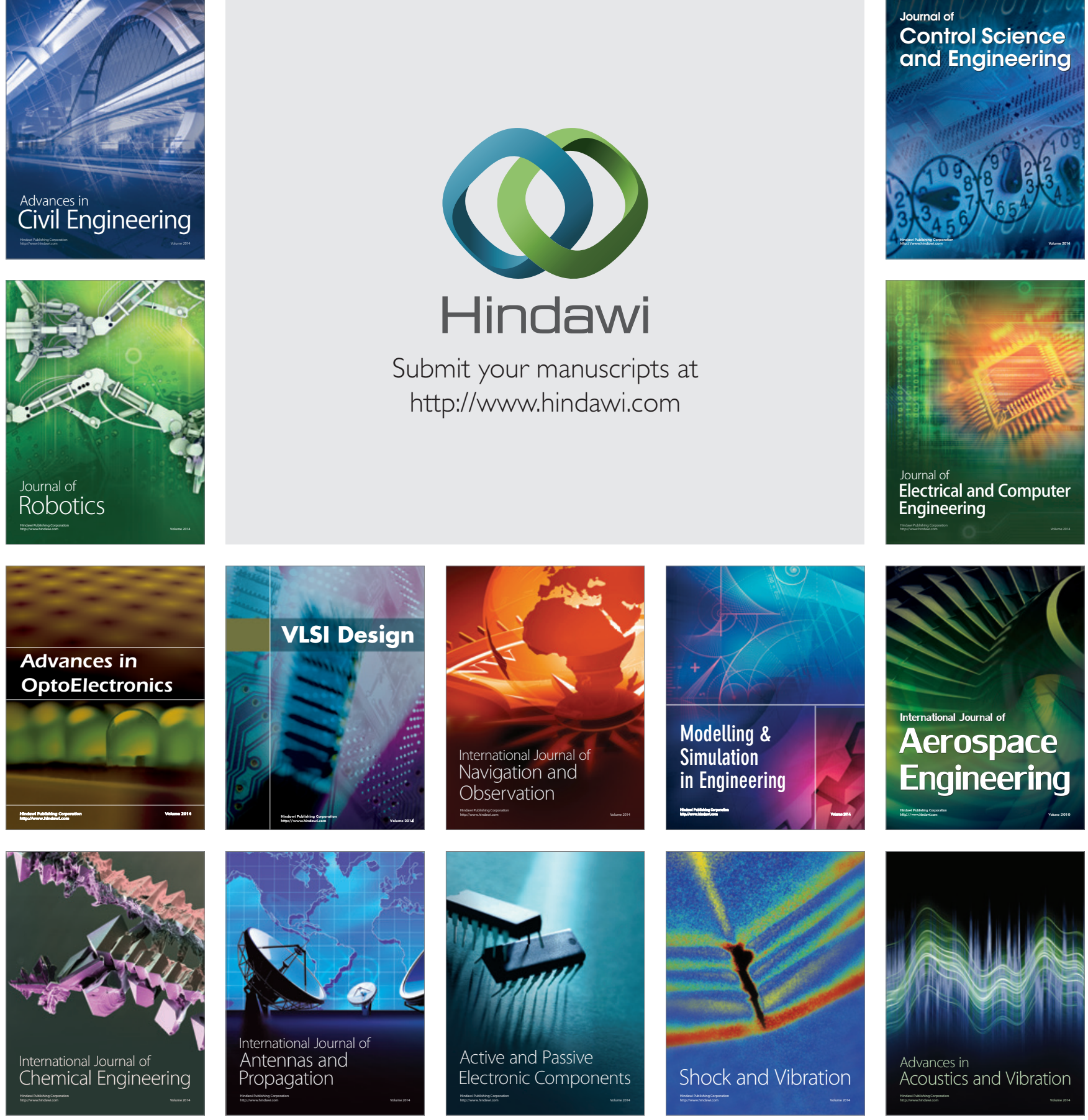